\title{
Homogenized couple stress model of optimal auxetic microstructures computed by topology optimization
}

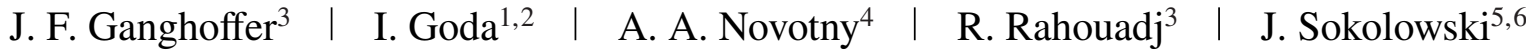

${ }^{1}$ LPMT, Université de Haute-Alsace, 11 rue Alfred Werner, 68093 Mulhouse Cedex, France

${ }^{2}$ Department of Industrial Engineering, Faculty of Engineering, Fayoum University, Fayoum 63514, Egypt

${ }^{3}$ LEMTA, Université de Lorraine. 2, CNRS, Avenue de la Forêt de Haye, BP 90161, 54505 Vandoeuvre Cedex, France

${ }^{4}$ Laboratório Nacional de Computação Científica LNCC/MCT, Coordenação de Matemática Aplicada e Computacional, Av. Getúlio Vargas 333, 25651-075 Petrópolis - RJ, Brasil

${ }^{5}$ Université de Lorraine, CNRS, Institute Elie Cartan, Nancy, UMR7502, BP 239, 54506

Vandoeuvre-Les-Nancy Cedex, France

${ }^{6}$ Systems Research Institute, Polish Academy of Sciences, Warsaw, Poland

\section{Correspondence}

J. F. Ganghoffer, LEMTA, Université de Lorraine. 2, Avenue de la Forêt de Haye, BP 90161 , 54505 Vandoeuvre Cedex, France.

Email: jean-francois.ganghoffer@univlorraine.fr
Auxetic materials and microstructures are attracting the attention of a growing community of researchers due to their unusual properties and high mechanical performances, in both the static and dynamic regimes. The topological derivative is used in this contribution to determine microstructures having the most negative in-plane mean Poisson's ratio. The auxetic nature of the computed microstructures is demonstrated by both numerical and real experiments performed over samples fabricated by additive printing. The effective mechanical properties of these auxetic structures have been computed in the framework of couple stress elasticity, allowing to identify both in-plane and out-of plane effective properties. The calculated classical moduli are found independent of the size of the window of analysis and are consequently effective coefficients. In contrast to this, the calculated in-plane bending moduli show a clear dependency on the auxetic cell size, whereas the out-of-plane bending moduli appear to be size-independent.

\section{K E Y W O R D S}

auxetic material, couple stress model, mechanical testing, microstructure design, poisson ratio, topological derivative

\section{1 | INTRODUCTION}

The emergence of additive manufacturing in combination with topology optimisation methods has led to the emergence of a new paradigm in the design of materials, coined material by design, in which the organization of matter plays a central role. The design of architectured and network materials requires to master and control microstructural effects, and consequently the inherent scale effects. It is well known that a great variety of non-standard behaviours can be obtained due to the network topology.

Materials with negative Poisson's ratio (abbreviated NPR in the sequel) were termed as Auxetic Materials (meaning antirubber) by Evans and Alderson; they have the property to expand in the lateral direction when stretched in the longitudinal direction. NPR materials have demonstrated rather unusual mechanical properties and to show exceptional performances, including an enhanced shear and indentation resistance, high fracture toughness, high energy dissipation ability and excellent dynamic crushing performance. NPR materials find potential applications in many fields, such as aerospace, protection against impact, or the biomedical sector. The effective properties of such auxetic materials are controlled by varying the topology of the repetitive unit cells within the structure.

The superiority of such auxetic materials ${ }^{[30]}$ is related to their heterogeneity, ${ }^{[24]}$ so that tailoring or optimizing the microstructure is a central issue in many research works, in order to fulfill certain macroscopic or mesoscopic performances. ${ }^{[28,29]}$ The design of auxetic structures has attracted many researchers. Conceptual designs of composite materials with Poisson's ratio close to -1 are presented in [15]. Design techniques using numerical approaches such as topology optimization started with the work 
of Sigmund, ${ }^{[22]}$ wherein a topology optimization framework for designing both $2 \mathrm{D}$ and 3D auxetic truss-based structures is presented. Different techniques are also adopted for auxetic structure design optimization. In [19], the authors study the topology design for auxetic structures using bi-directional evolutionary structural optimization. In [29], the authors develop a new level-set based topology optimization method for the computational design of multimaterial metamaterials with exotic thermomechanical properties. In [12] a topology optimization methodology of framed structures to design a planar periodic structure that exhibits negative Poisson's ratio has been proposed. Using both mathematical and numerical methods, isotropic design optimization for auxetic material is studied recently by [5]. Recent work by [27] introduced smoothed petal-shaped auxetic structures, where the hinges are replaced by smoothed connections. The optimized designs are shown to achieve low negative Poisson's ratios, while the difficulties of manufacturing the hinges are avoided.

Starting historically from the SIMP method (Solid Isotropic Material Penalization) in the late eighties [2] (see also [3]), different topology optimization methods have been developed in the literature, including the bubble method, ${ }^{[6]}$ the bidirectional structural optimization technique (BESO) presented in [11], and the topological derivative exposed in [7] and [8]. In [25] and [28] a new topological shape optimization method for systematic computational design of a type of mechanical metamaterials with negative Poisson's ratios has been developed, which integrates the numerical homogenization approach into a powerful parametric level set method. More recently, in [25] a level set based method for topology optimization of both single and multiple-material Negative Poisson's Ratio metamaterials has been proposed. In the literature, nonlinear materials with prescribed stress-strain response or prescribed Poisson's ratio are designed in [26], relying on the SIMP method to interpolate material properties between full material properties or void. The authors analyze the optimization of the material design under finite deformation. The employed topology optimization method allows a systematic virtual design of materials subjected to tensile tests, by minimizing the errors between the actual and prescribed mechanical properties in a given axial strain range. In [4], a family of 3D auxetic cubic lattices is proposed, which presents a unidirectional Poisson's ratio close to the stability limit of -1 ; the obtained value of Poisson's ratio is direction dependent and function of the ratio of the tensile to bending stiffness of the truss elements. A specific structure has been tailored to give an isotropic behavior with Poisson's ratio close of even less than -1 along certain directions. Elastic metamaterials with Poisson's ratio close to the lower bound of -1 for isotropic materials have been computed based on a parametric level set method in [26]. An optimization framework for 3D porous solids is presented in [24], leading to the computation of microstructures having optimal effective elasticity properties, especially minimum Poisson's ratio. As outlined in [24], the topological derivative based approach does not involve a regularization parameter, which makes it more attractive compared to the SIMP method.

The objective of the present work is to determine numerically microstructures presenting an optimal auxetic behavior in a planar situation, relying on the topological derivative as a mathematical optimization tool. Thereby, we shall combine homogenization with topology optimization to design optimally designed metamaterials showing an auxetic behavior. The proposed methodology is systematic and goes well beyond the heuristic most often employed in the literature to design such microstructures. Both numerical experiments designed to evaluate an effective constitutive couple stress model and real experiments done on samples of the identified auxetic microstructures shall be performed.

The novelty of the manuscript consists accordingly in the design, testing and numerical evaluation of the effective moduli of auxetic periodic microstructures took from [1]. We shall design and manufacture 3D periodically microporous structures (with planar periodicity) based on optimal unit cells using BLENDER, CATIA, and ABAQUS software environments and additive printing technology, and next identify experimentally and numerically the effective constitutive coefficients of an enriched constitutive model accounting for the local microrotations and strain gradients that develop within the tested samples.

The generalized continua that may emerge when substituting the computed initially discrete network by an effective continuum provide a description of the kinematics and constitutive response showing microstructural and scale effects which are richer in comparison to Cauchy classical elasticity; these microstructural effects are potentially a source of novel unexpected effects like Poisson's ratio outside the isotropic limits of conventional structures (like negative Poisson's ratio), which make them suitable for a wide range of potential applications in which these unusual behaviours will have a great benefit. There are many discussions on the use of generalized continua to model auxetic microstructures in the literature. ${ }^{[13,16,18,21,28,30]}$

Although such generalized continuum media have been widely used to regularize ill-posed boundary value problems and reduce the computational cost inherent to large repetitive structures, they have never been employed to produce new microstructures.

Novel aspects presented in the present paper are the following:

- Optimal orthotropic auxetic microstructures are computed based on the topological derivative;

- A couple stress model is identified for the designed auxetic microstructures;

- The effective Poisson's ratio predicted by topology optimization is validated by numerical simulations and real measurements. 


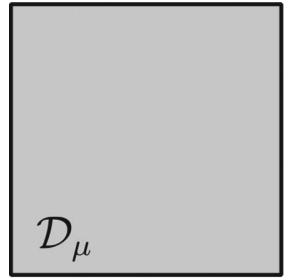

(a)

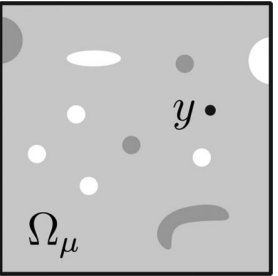

(b)

F I G U R E 1 Hold-all RVE domain $\mathcal{D}_{\mu}$ (a) and RVE domain $\Omega_{\mu}$ (b)

The evaluation of the topological derivative of the homogenized elasticity tensor is done in Section 2, leading to the closed form expression of the topological derivative of the average in-plane Poisson's ratio. Tensile experiments for the auxetic samples fabricated by additive printing are performed in Section 3 to evaluate their relevant mechanical properties, especially Poisson's ratio. In Section 4, we compute the homogenized linear response of the optimal auxetic network material, relying on an analysis performed over a repetitive unit cell at a mesoscopic level, intermediate between the microscopic and continuum scales. We conclude in Section 5 with a summary of the main achievements of the present work and a few perspectives.

\section{2 । COMPUTATION OF AUXETIC MICROSTRUCTURES BASED ON THE TOPOLOGICAL DERIVATIVE CONCEPT}

In order to set the stage, we synthetically recall the notion of topological derivative which is the topology optimization method employed in the present work to determine optimal auxetic microstructures. The topological derivative represents the first term of the asymptotic expansion of a given shape functional with respect to the small parameter which measures the size of singular domain perturbations, such as holes, inclusions, source-terms and cracks. This relatively new concept was introduced in the fundamental paper ${ }^{[23]}$ and has been successfully applied to many relevant fields such as shape and topology optimization, inverse problems, imaging processing, multiscale material design and mechanical modeling including damage and fracture evolution phenomena. See, for instance, the book by Novotny \& Sokołowski, 2013. ${ }^{[17]}$ In the context of multiscale material design, the topological derivative obtained in [8] represents the sensitivity of the homogenized elasticity tensor to the nucleation of a circular hole within the Representative Volume Element (RVE) whose domain is denoted by $\mathcal{D}_{\mu}$, with boundary $\partial \mathcal{D}_{\mu}$. More precisely, the homogenized elasticity tensor $\mathbb{C}$, whose components written in terms of the orthonormal basis $\left\{e_{i}\right\}$, for $i=1,2$, of the Euclidean space, can be defined as

$$
(\mathbb{C})_{i j k l}=\frac{1}{V_{\mu}} \int_{\Omega_{\mu}} \sigma_{\mu}\left(u_{\mu}^{k l}\right) \cdot \varepsilon_{\mu}\left(u_{\mu}^{i j}\right)
$$

where $\Omega_{\mu} \subset \mathcal{D}_{\mu}$ and $V_{\mu}$ denotes the total volume of the RVE, namely $V_{\mu}=\left|D_{\mu}\right|$. See Figure 1 . The canonical microscopic displacement field $u_{\mu}^{k l}(y)$, with $y \in \Omega_{\mu}$, may be decomposed into a sum

$$
u_{\mu}^{k l}(y):=u+\left(e_{k} \otimes e_{l}\right) y+\widetilde{u}_{\mu}^{k l}(y)
$$

of a constant (rigid) RVE displacement coinciding with the macroscopic displacement field $u$, a linear field $\left(e_{k} \otimes e_{l}\right) y$, and a canonical microscopic displacement fluctuation field $\widetilde{u}_{\mu}^{k l}(y)$ solution of [20]

$$
\widetilde{u}_{\mu}^{k l} \in \mathcal{V}_{\mu}: \int_{\Omega_{\mu}} \sigma_{\mu}\left(\widetilde{u}_{\mu}^{k l}\right) \cdot \varepsilon_{\mu}(\eta)+\int_{\Omega_{\mu}} \mathbb{C}_{\mu}\left(e_{k} \otimes_{s} e_{l}\right) \cdot \varepsilon_{\mu}(\eta)=0 \quad \forall \eta \in \mathcal{V}_{\mu},
$$

where $\otimes_{S}$ denotes the symmetric tensor product between vectors and $\eta$ is the test function living in $\mathcal{V}_{\mu}$. The virtual strain tensor $\varepsilon_{\mu}(\eta)$ is given by the symmetric part of the gradient of $\eta$. We assume that the microscopic stress tensor field $\sigma_{\mu}\left(\widetilde{u}_{\mu}^{k l}\right)$ satisfies

$$
\sigma_{\mu}\left(\widetilde{u}_{\mu}^{k l}\right)=\mathbb{C}_{\mu} \varepsilon_{\mu}\left(\widetilde{u}_{\mu}^{k l}\right),
$$


where $\mathbb{C}_{\mu}$ is the microscopic constitutive tensor, which in terms of the Lamé's coefficients $\mu$ and $\lambda$ is given by

$$
\mathbb{C}_{\mu}=2 \mu \rrbracket+\lambda \mathrm{I} \otimes \mathrm{I},
$$

with I and $\square$ used to denote the second and fourth order identity tensors, respectively. Moreover, the space $\mathcal{V}_{\mu}$ of kinematically admissible displacement fluctuations is defined as follows

$$
\mathcal{V}_{\mu}:=\left\{\varphi \in \mathcal{V}_{\mu}: \varphi\left(y^{+}\right)=\varphi\left(y^{-}\right) \quad \forall\left(y^{+}, y^{-}\right) \in \mathfrak{P}\right\},
$$

where $\mathfrak{P}$ is the set of pairs of points, defined by a one-to-one periodicity correspondence, lying on opposing sides of the RVE boundary $\partial \mathcal{D}_{\mu}$. Finally, the minimally constrained space of kinematically admissible displacements $V_{\mu}$ is defined as

$$
\mho_{\mu}:=\left\{\varphi \in H^{1}\left(\Omega_{\mu}\right): \int_{\Omega_{\mu}} \varphi=0, \int_{\partial D_{\mu}} \varphi \otimes_{S} n=0\right\} .
$$

where $n$ is the outward unit normal to the boundary $\partial \mathcal{D}_{\mu}$ and $\otimes_{S}$ denotes the symmetric tensor product between vectors.

Since each component of the homogenized elasticity tensor is defined by the energy based functional (2.1), the associated topological derivative with respect to the nucleation of a circular hole at an arbitrary point of $\Omega_{\mu}$ is given by [8]

$$
\mathcal{T}_{\mu}(y)=-\frac{1}{V_{\mu}} \mathbb{P}_{\mu} \sigma_{\mu}\left(u_{\mu}^{i j}(y)\right) \cdot \varepsilon_{\mu}\left(u_{\mu}^{k l}(y)\right), \quad \forall y \in \Omega_{\mu},
$$

with the polarization tensor $\mathbb{P}_{\mu}$ defined as

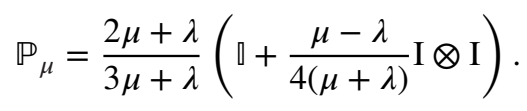

From the result given by (2.8), we can recognize a fourth order tensor field over $\Omega_{\mu}$ that represents the sensitivity of the macroscopic elasticity tensor $\mathbb{C}$ to topological microstructural changes resulting from the insertion of a circular hole within the RVE. Therefore, the topological derivative of the homogenized elasticity tensor reads

$$
\mathbb{D}_{\mu}(y)=-\frac{1}{V_{\mu}} \mathbb{P}_{\mu} \sigma_{\mu}\left(u_{\mu}^{i j}(y)\right) \cdot \sigma_{\mu}\left(u_{\mu}^{k l}(y)\right) e_{i} \otimes e_{j} \otimes e_{k} \otimes e_{l},
$$

where the fields $u_{\mu}^{i j}$ come out from the solutions to (2.3) for the unperturbed RVE domain $\Omega_{\mu}$ together with the additive decomposition (2.2).

Expression (2.10) allows the exact topological derivative of any differentiable function of $\mathbb{C}$ be calculated through the direct application of the conventional rules of differential calculus. That is, any such a function $\Psi(\mathbb{C})$ has exact topological derivative of the form

$$
\mathcal{T}_{\mu}=\left\langle D \Psi(\mathbb{C}), \mathbb{D}_{\mu}\right\rangle,
$$

with the brackets $\langle\cdot, \cdot\rangle$ denoting the appropriate product between the derivative of $\Psi$ with respect to $\mathbb{C}$ and the topological derivative $\mathbb{D}_{\mu}$ of $\mathbb{C}$. In particular, let $\varphi_{1}, \varphi_{2} \in \mathbb{R}^{2} \times \mathbb{R}^{2}$ be any pair of second order tensors. Then, for a function $\Psi(\mathbb{C})$ of the form

$$
\Psi(\mathbb{C}):=\frac{\mathbb{C}^{-1} \varphi_{1} \cdot \varphi_{2}}{\mathbb{C}^{-1} \varphi_{1} \cdot \varphi_{1}}+\frac{\mathbb{C}^{-1} \varphi_{2} \cdot \varphi_{1}}{\mathbb{C}^{-1} \varphi_{2} \cdot \varphi_{2}}
$$

the corresponding topological derivative is given by

$$
\begin{aligned}
\mathcal{T}_{\mu}= & -\frac{\left(\mathbb{C}^{-1} \mathbb{D}_{\mu} \mathbb{C}^{-1}\right) \varphi_{1} \cdot\left[\left(\mathbb{C}^{-1} \varphi_{1} \cdot \varphi_{1}\right) \varphi_{2}-\left(\mathbb{C}^{-1} \varphi_{1} \cdot \varphi_{2}\right) \varphi_{1}\right]}{\left(\mathbb{C}^{-1} \varphi_{1} \cdot \varphi_{1}\right)^{2}} \\
& -\frac{\left(\mathbb{C}^{-1} \mathbb{D}_{\mu} \mathbb{C}^{-1}\right) \varphi_{2} \cdot\left[\left(\mathbb{C}^{-1} \varphi_{2} \cdot \varphi_{2}\right) \varphi_{1}-\left(\mathbb{C}^{-1} \varphi_{2} \cdot \varphi_{1}\right) \varphi_{2}\right]}{\left(\mathbb{C}^{-1} \varphi_{2} \cdot \varphi_{2}\right)^{2}}
\end{aligned}
$$

where $\mathbb{C}^{-1}$ is used here to denote the compliance tensor $\mathbb{S}$. 


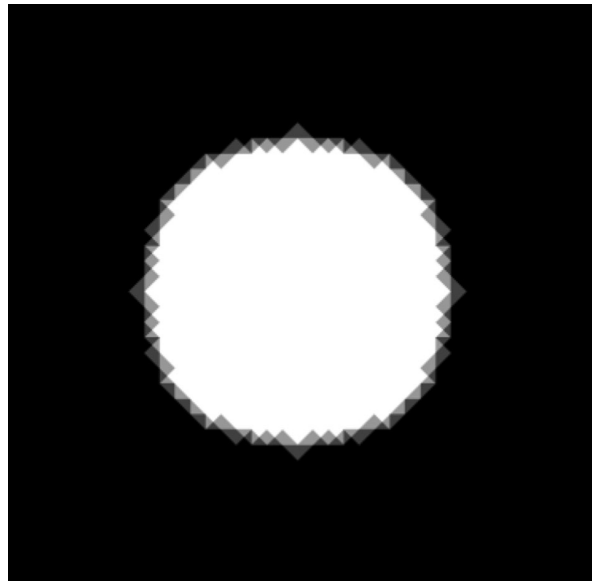

F I G URE 2 Initial guess of the porous microstructure $\Omega_{\mu}$

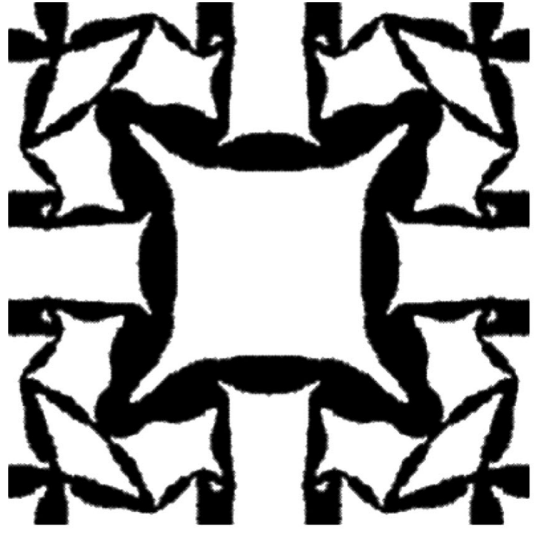

(a)

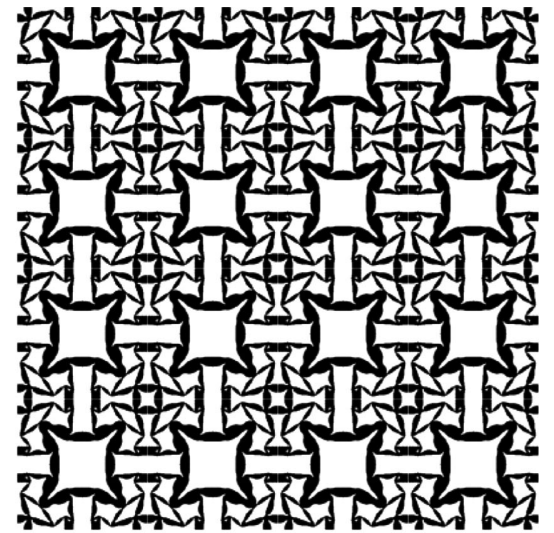

(b)

F I G U R E 3 Optimized auxetic microstructure (a) and periodic auxetic structure (b) obtained from [1]

The topology optimization problem we are dealing with consists in minimizing $\Psi(\mathbb{C})$ with respect to $\Omega_{\mu} \subset D_{\mu}$. Mathematically, it can be written as follows

$$
\underset{\Omega_{\mu} \subset D_{\mu}}{\operatorname{Minimize}} \Psi(\mathbb{C})
$$

where $\Psi(\mathbb{C})$ is defined in (2.12), whose dependence with respect to the design domain $\Omega_{\mu}$ comes out from the definition of the homogenized elasticity tensor $\mathbb{C}$ given by $(2.1)$.

Let us now consider one RVE into two spatial dimensions given by a unit square $\mathcal{D}_{\mu}=(0,1) \times(0,1)$. The Young's modulus and the Poisson's ratio of the bulk material are respectively set as $E_{\mu}=1725 M P a$ and $\nu_{\mu}=0.3$. The initial guess is given by a porous microcell $\Omega_{\mu}$ as shown in Figure 2. By setting $\varphi_{1}=e_{1} \otimes e_{1}$ and $\varphi_{2}=-e_{2} \otimes e_{2}$ in (2.12), the resulting function $\Psi(\mathbb{C})$ represents twice the arithmetic mean of both in-plane Poisson's ratio, expressing as

$$
\Psi(\mathbb{C}):=-\frac{\left(\mathbb{C}^{-1}\right)_{1122}}{\left(\mathbb{C}^{-1}\right)_{1111}}-\frac{\left(\mathbb{C}^{-1}\right)_{2211}}{\left(\mathbb{C}^{-1}\right)_{2222}} .
$$

The optimal value of the average in-plane Poisson ratio is -0.82 , and the obtained optimized auxetic microstructure by to [1] is presented in Figure 3(a), while the resulting periodic auxetic structure is shown in Figure 3(b); due to the adopted definition of Poisson's ratio, the obtained microstructure is orthotropic. For more details on the numerical implementation see [1], where the adopted topology design algorithm is presented. We remark however that the result presented in Figure 3 is just a local minimizer. Indeed, there is a lack of sufficient optimality conditions for such topology optimization problems, thus no global minimizer can usually be obtained. 


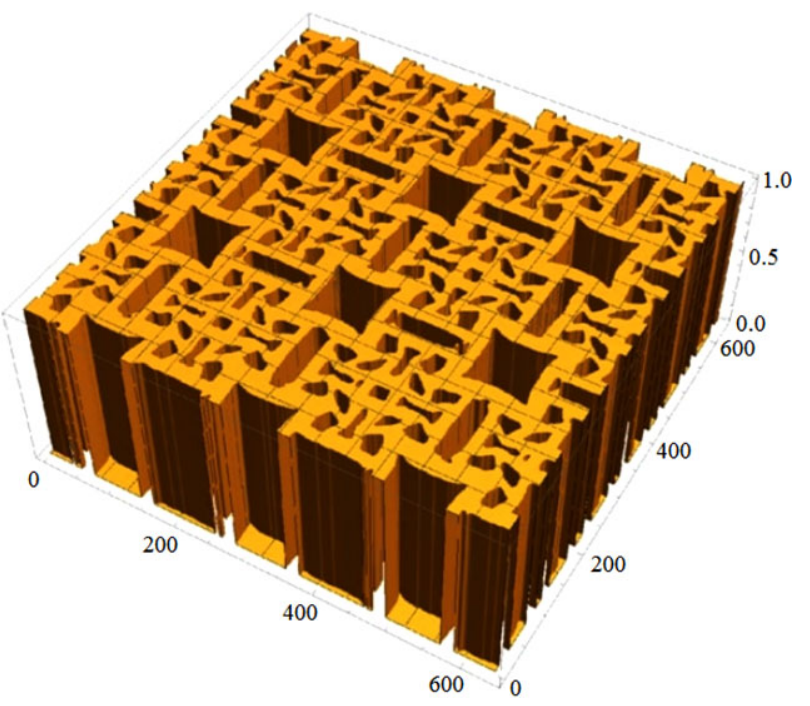

F I G U R E 4 Auxetic samples produced by additive printing

\section{3 | MEASUREMENTS OF THE MECHANICAL RESPONSE OF THE AUXETIC SAMPLES}

A 3D structure based on the unit cell having the microstructure computed by the topological derivative exposed in previous section has been generated using the software BLENDER, starting from the quadrilateral shapes in the plane, and which have further been extruded to get a 3D geometry. The generated images have next been exported to CATIA to produce an initial geometry, which has finally been exported to ABAQUS. Based on this geometry, samples made of Acrylonitrile Butadiene Styrene (ABS in short cut) have been produced by additive printing, as shown on Figure 4.

The fabricated structures are designed as plates with $216 \mathrm{~mm}$ length (this defines the $x$ direction), 120 width, and a uniform thickness of $4 \mathrm{~mm}$ (the thickness of each newly added layer is $0.3 \mathrm{~mm}$ ). The Young's modulus of the bulk material was measured by tensile testing of homogeneous plates as $E_{\mu}=1725 \mathrm{MPa}$, and Poisson's ratio measured as $v_{\mu}=0.3$. The samples have been tested in uniaxial tension along the $x$ direction with a MTS2/M10kN standard tensile machine, at a constant velocity of $5 \mathrm{~mm} / \mathrm{min}$, up to fracture. Uniaxial measurements of the auxetic samples lead to a measured apparent Young's modulus in the tensile $x$ direction equal to $E_{x}=30 \mathrm{MPa}$, a value much lower than the bulk material tensile modulus, reflecting the impact of the microporosity. We performed overall 5 measurements for different specimen, and obtained a high correlation coefficient of 0.95. Since this is indicative of a small dispersion of measurements, we can judge that average value of the measured effective tensile modulus is representative of the measured specimen properties.

The important difference between the responses of the homogeneous plate and the auxetic plate can be explained by the inherent microstructure, which can be accounted in a global manner by the reduced section of material supporting the load within the sample. The normalized corrected section $S(x) / S_{0}$ (by the section of the homogeneous plate) evolution versus the axial variable $x$ shown in Figure 3(b) is pictured on Figure 5. The corrected section is shown to exhibit strong variations by a factor close to 10, as shown in Figure 5, with the minimum ratio $S(x) / S_{0}=0.1$.

Normalizing the force-displacement measured response for the homogeneous plate by the fraction of section occupied by the auxetic structure (adopting as the corrected section the minimum value recorded on Figure 5) leads to a response close to that of the homogeneous auxetic sample, as shown on Figure 6.

We have continuously recorded the change of configuration of the tested samples, allowing to evaluate the evolution of Poisson's ratio for a central window of analysis versus displacement on Figure 7; the effective in-plane Poisson's ratio $v_{x y}$ is computed as (minus) the ratio of logarithmic strains measured in the transverse to axial directions.

Poisson's ratio is obtained as the slope of the interpolating line, in the range $[-0.82,-0.77]$, accounting for the obtained variability of measurements. The high correlation coefficient $(0.91)$ of the obtained linear interpolation indicates a nearly constant Poisson's value in the considered small strains interval of the testing (below $2.5 \%$ ). The next section is devoted to the numerical evaluation of the effective in-plane and bending moduli of the previously tested samples. 


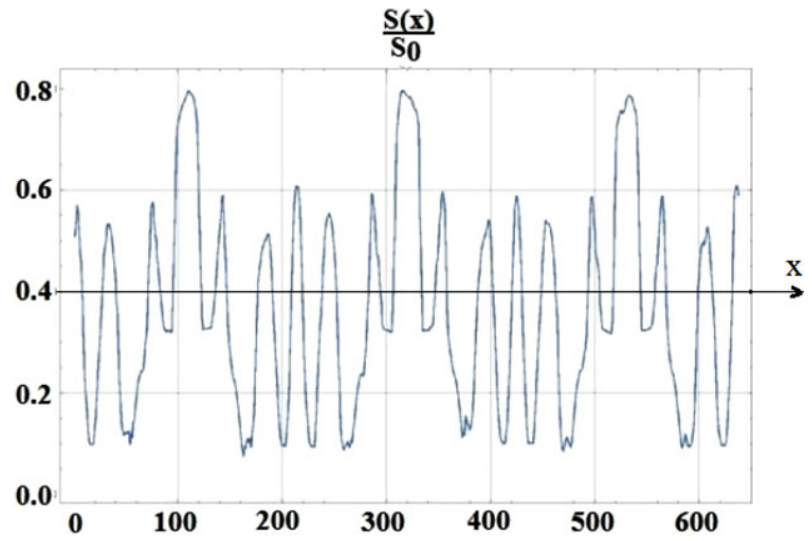

F I G U R E 5 Normalized section occupied by the material within the auxetic sample versus coordinate $x$. The scalar function $S(x)$ is the corrected section, while $S_{0}$ is the section of the homogeneous plate without microstructure

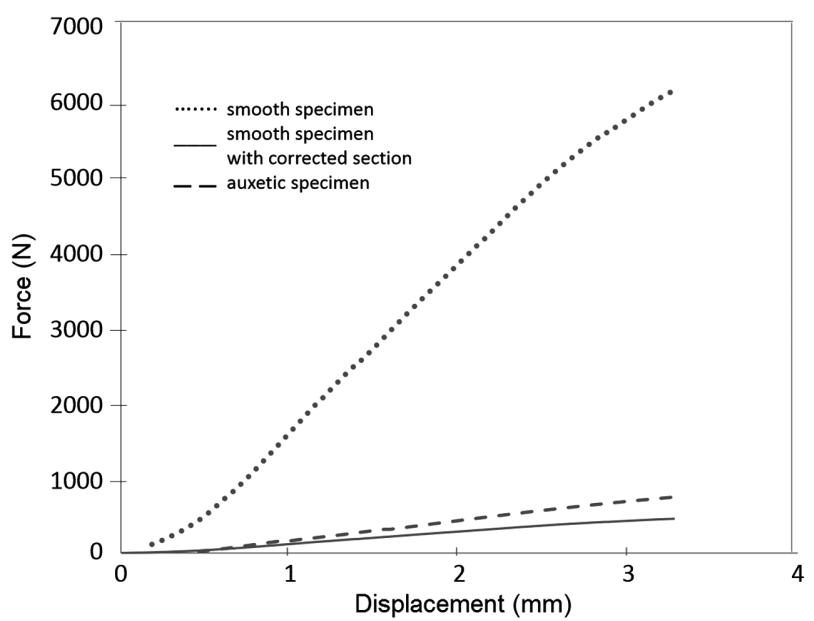

F I G U R E 6 Comparison of the tensile response of the homogeneous plate with the normalized response of the auxetic sample

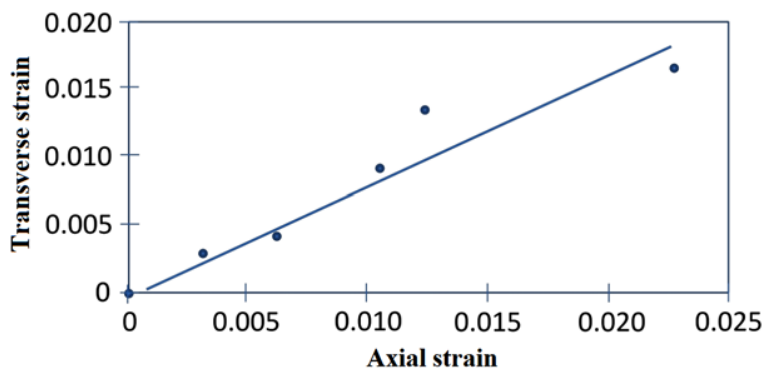

F I G U R E 7 Evolution of the transverse strain versus axial strain and measurement of Poisson's ratio $v_{x y}$

\section{4 | COMPUTATION OF THE CLASSICAL AND COUPLE STRESS MODULI}

Classical continuum mechanics may not be the most appropriate macroscopic description of such porous auxetic structures because higher order effects - absent in classical theories - are required to afford the transition from the discrete to an equivalent continuum description. Performing a FE analysis of the displacement field at the microstructural level for a unit cell under traction (along the vertical direction) reveals the existence of pronounced rotations of internal domains, as illustrated on Figure 8.

These local rotations are associated to micro moments that do not balance each other; their existence motivates adopting a couple-stress theory at the mesoscopic level. Figure 8 shows the deformed shape of the unit cell superposed onto the initial 


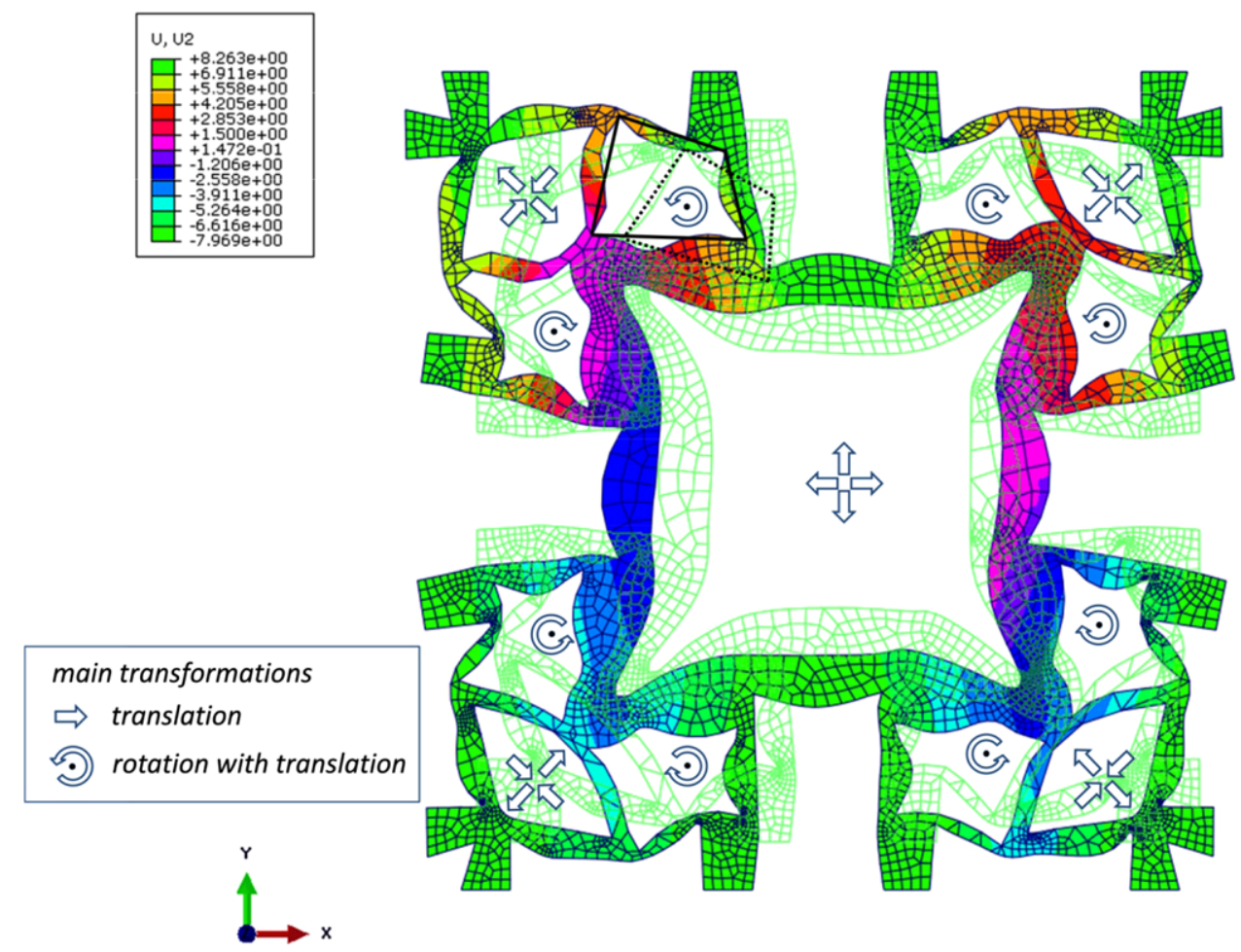

F I G U R E 8 Kinematics of the unit cell subjected to a tensile loading along the vertical direction. The local kinematics is indicated by arrows

undeformed shape shown with light colors. The local rotation field shown on Figure 8 is further responsible for the auxetic effects, since the two domains symmetrical with respect to the diagonal rotate in opposite directions, so that the unit cell expands in the horizontal unloaded direction while loaded in the vertical direction. This numerical evidence of pronounced rotation effects at the microscopic scale motivates the use of a couple-stress model.

In the micropolar continuum, the deformation is described by the displacement vector with components $u_{i}$ and an independent rotation vector, whereas in the couple stress theory, the rotation vector is not independent of the displacement vector: the rotation vector with components $\phi_{x}, \phi_{y}, \phi_{z}$ in a general $3 \mathrm{D}$ situation is related to the displacement gradients as:

$$
\begin{aligned}
& \phi_{x}=\frac{1}{2}\left(\frac{\partial u_{z}}{\partial y}-\frac{\partial u_{y}}{\partial z}\right), \\
& \phi_{y}=\frac{1}{2}\left(\frac{\partial u_{x}}{\partial z}-\frac{\partial u_{z}}{\partial x}\right), \\
& \phi_{z}=\frac{1}{2}\left(\frac{\partial u_{y}}{\partial x}-\frac{\partial u_{x}}{\partial y}\right) .
\end{aligned}
$$

The kinematic variables to be taken into account in the framework of linear couple-stress theory of elasticity are the strain $\varepsilon_{i j}$ and the gradient of the microrotation, the tensor with components $\chi_{i j}$; they are defined, respectively, as

$$
\varepsilon_{i j}=\frac{1}{2}\left(u_{i, j}+u_{j, i}\right)=\varepsilon_{j i} \quad \text { and } \quad \chi_{i j}=\frac{1}{2}\left(\phi_{i, j}+\phi_{j, i}\right)=\chi_{j i}, \quad i, j=x, y, z .
$$

The strain tensor $\varepsilon_{i j}$ is symmetrical, with components depending upon the displacements gradients as:

$$
\begin{aligned}
& \varepsilon_{x x}=\frac{\partial u_{x}}{\partial x} ; \varepsilon_{y y}=\frac{\partial u_{y}}{\partial y} ; \varepsilon_{z z}=\frac{\partial u_{z}}{\partial z} ; \\
& \varepsilon_{x y}=\varepsilon_{y x}=\frac{1}{2}\left(\frac{\partial u_{y}}{\partial x}+\frac{\partial u_{x}}{\partial y}\right) ;
\end{aligned}
$$




$$
\begin{aligned}
& \varepsilon_{x z}=\varepsilon_{z x}=\frac{1}{2}\left(\frac{\partial u_{z}}{\partial x}+\frac{\partial u_{x}}{\partial z}\right) ; \\
& \varepsilon_{y z}=\varepsilon_{z y}=\frac{1}{2}\left(\frac{\partial u_{z}}{\partial y}+\frac{\partial u_{y}}{\partial z}\right) .
\end{aligned}
$$

The couple-stress curvature tensor $\chi_{i j}$ is an asymmetric tensor; its independent components are explicitly written from the gradient of the microrotation vector as

$$
\begin{aligned}
& \chi_{x x}=\frac{1}{2}\left(\frac{\partial^{2} u_{z}}{\partial x \partial y}-\frac{\partial^{2} u_{y}}{\partial z \partial x}\right) ; \chi_{x y}=\frac{1}{2}\left(\frac{\partial^{2} u_{x}}{\partial z \partial x}-\frac{\partial^{2} u_{z}}{\partial x^{2}}\right) ; \chi_{x z}=\frac{1}{2}\left(\frac{\partial^{2} u_{y}}{\partial x^{2}}-\frac{\partial^{2} u_{x}}{\partial x \partial y}\right) ; \\
& \chi_{y y}=\frac{1}{2}\left(\frac{\partial^{2} u_{x}}{\partial y \partial z}-\frac{\partial^{2} u_{z}}{\partial x \partial y}\right) ; \chi_{y x}=\frac{1}{2}\left(\frac{\partial^{2} u_{z}}{\partial y^{2}}-\frac{\partial^{2} u_{y}}{\partial y \partial z}\right) ; \chi_{y z}=\frac{1}{2}\left(\frac{\partial^{2} u_{y}}{\partial x \partial y}-\frac{\partial^{2} u_{x}}{\partial y^{2}}\right) ; \\
& \chi_{z z}=\frac{1}{2}\left(\frac{\partial^{2} u_{y}}{\partial x \partial z}-\frac{\partial^{2} u_{x}}{\partial y \partial z}\right) ; \chi_{z x}=\frac{1}{2}\left(\frac{\partial^{2} u_{z}}{\partial y \partial z}-\frac{\partial^{2} u_{y}}{\partial z^{2}}\right) ; \chi_{z y}=\frac{1}{2}\left(\frac{\partial^{2} u_{x}}{\partial z^{2}}-\frac{\partial^{2} u_{z}}{\partial x \partial z}\right)
\end{aligned}
$$

For solids endowed with central symmetry (an assumption that will be made here in a realistic manner), the constitutive equations of the effective couple stress continua can be expressed in a compact form as follows:

$$
\{\sigma\}=[C]\{\varepsilon\} \text { and }\{m\}=[D]\{\chi\}
$$

with the stiffness matrix $[C]$ relating stresses to strains and the curvature tensor $[D]$ relating couple stresses to curvatures.

The stress and couple-stress tensors for the effective 3D couple stress continuum can be defined in vector format as

$$
\begin{aligned}
& \{\sigma\}=\left\{\sigma_{x x}, \sigma_{y y}, \sigma_{z z}, \sigma_{x y}, \sigma_{y z}, \sigma_{x z}\right\}^{\top}, \\
& \{m\}=\left\{m_{x x}, m_{y y}, m_{z z}, m_{x y}, m_{y x}, m_{y z}, m_{z y}, m_{x z}, m_{z x}\right\}^{\top},
\end{aligned}
$$

wherein the three components $m_{x x}, m_{y y}, m_{z z}$ are the torsion couple-stresses and the six components $m_{x y}, m_{y x}, m_{y z}, m_{z y}, m_{x z}, m_{z x}$ are the bending couple stresses.

The main purpose of this section is to determine the effective constitutive coefficients of the couple-stress continuum from the response of the numerically tested auxetic samples. Note that although the procedures of computing all couple stress components (twisting and bending) are exposed, we would presently focus on the bending curvatures behaviors of both in-plane and outof-plane situations for the underlying auxetic structures. We design different boundary conditions for the determination of the components of the rigidity constants over the domain $\Omega_{\mu}$ in Figure 9. In each case, we force the RVE in a 3D context to bear the designed specific deformations $\varepsilon_{i j}$ and curvatures $\chi_{i j}$, and compute numerically (by finite elements) the total elastic strain energy $U_{\mathrm{RVE}}$ stored in the RVE under the corresponding boundary conditions. The adopted numerical procedure relies on equating the total elastic strain energy stored in the RVE to the energy of an equivalent homogeneous couple-stress continuum, thus it holds as a consequence of Hill-Mandel lemma extended to the present couple stree continuum the following equality:

$$
U_{\mathrm{RVE}}=U_{\text {couple-stress }}=\frac{V_{\mu}}{2}\left(\varepsilon_{i j} C_{i j k l} \varepsilon_{k l}+\chi_{i j} D_{i j k l} \chi_{k l}\right),
$$

where $V_{\mu}=\left|\Omega_{\mu}\right|$ is the volume of the RVE. The left-hand side in (4.8) is the total elastic strain energy stored in the RVE of the auxetic, which is evaluated thanks to FE computations performed over the representative unit cell. The right-hand side is the energy of the effective couple-stress continuum, which is a function of the volume-average strains $\varepsilon_{i j}$ and curvatures $\chi_{i j}$ of the unit cell, and involving the effective couple-stress moduli, which are to be identified. We aim at investigating the effect of scale size, therefore we choose different sample sizes involving the repetition of the smallest unit cell to form different unit cell sizes as shown in Figure 9. Since our objective is to study scale effects, then we are currently exploring how the elastic constants relying on couple stress continua within the elementary cell change when we increase the size of the repeated unit cell.

The developed numerical homogenization consists in determining the overall effective couple-stress elastic coefficients of the auxetic structure samples, relying on a finite element discretization of the RVE geometry. This is performed using mixed traction and displacement boundary conditions involving applied displacements and tractions. The applied loadings include 


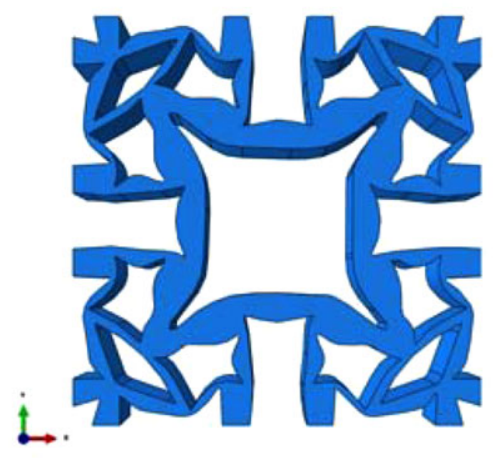

(a)

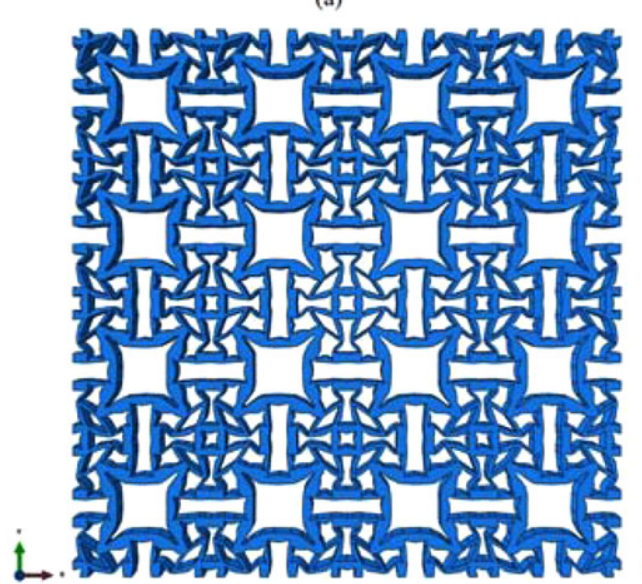

(c)

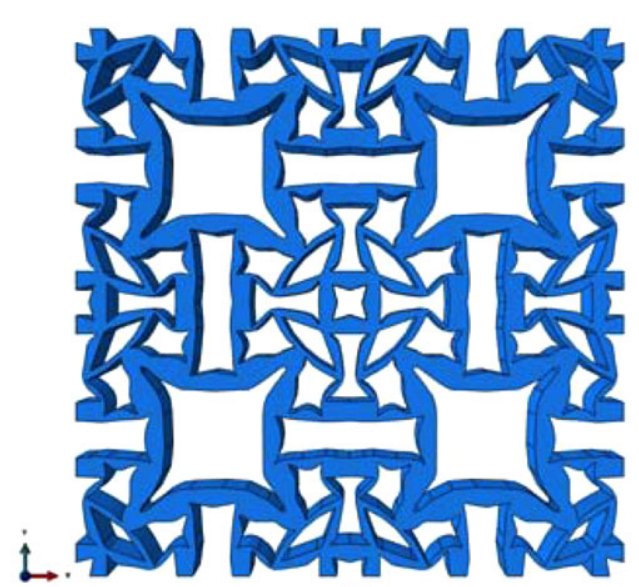

(b)

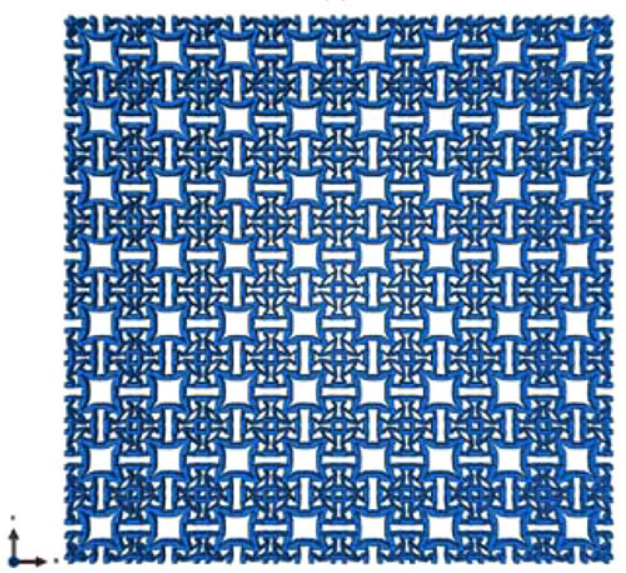

(d)

F I G U R E 9 Four different 3D periodic model representation of auxetic structures with (a) a single unit cell denoted $1 \times 1$, (b) $2 \times 2$ unit cells, (c) $4 \times 4$ unit cells, and (d) $8 \times 8$ unit cells

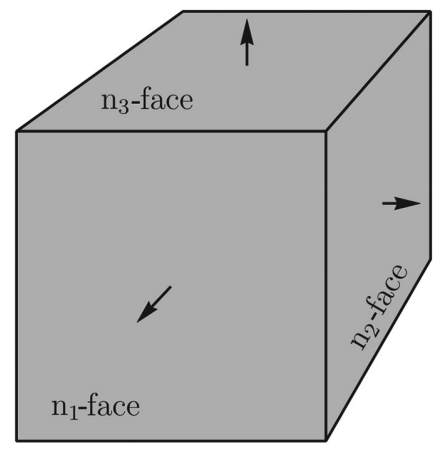

F I G U R E 10 The oriented faces $n_{1}$-face, $n_{2}$-face, and $n_{3}$-face

uniaxial extension tests, biaxial extension tests, in-plane and out-of plane shear tests to evaluate stiffness matrix components [C] and uniaxial twist (or torsion) tests, biaxial twist tests, in plane and out-of plane curvature (or bending moment) tests to evaluate the stiffness matrix components $[D]$. For each loading case, we compute by the FE method within the ABAQUS environment the total strain energy stored in the RVE and then equate this energy with the energy of an equivalent homogeneous couple-stress continuum, as previously explained. Since the auxetic samples are made from ABS, the isotropic bulk mechanical properties of the auxetic unit cells wall in the FE simulations are taken as $E_{\mu}=1725 \mathrm{MPa}$ and Poisson's ratio $v_{\mu}=0.3$. The methodology for computing the effective moduli involves the application of elementary unit strains and curvatures onto the RVEs. One has to be aware that this procedure is only for the purpose of computing moduli, but it has nothing to do with real tests, which should be limited to a strain or curvature range for which the auxetic structures remain elastic. Following the sketch in Figure 10, to evaluate the components of the stress stiffness tensor $[C]$, we conduct nine elementary tests, described in the sequel. 
(1) Uniaxial extension for $C_{11}$ : we apply a uniform strain $\varepsilon_{x x}=1$ and shear stress $\sigma_{x y}=\sigma_{y z}=\sigma_{x z}=0$ on the unit cell's boundary, the corresponding boundary conditions are then written as

$$
\begin{array}{lll}
u_{x}=x, \sigma_{x y} n_{1}=0, \sigma_{x z} n_{1}=0 & \text { on } & \mathrm{n}_{1} \text {-face, } \\
u_{y}=0, \sigma_{x y} n_{2}=0, \sigma_{y z} n_{2}=0 & \text { on } & \mathrm{n}_{2} \text {-face, } \\
u_{z}=0, \sigma_{x z} n_{3}=0, \sigma_{y z} n_{3}=0 & \text { on } & \mathrm{n}_{3} \text {-face, }
\end{array}
$$

which leads to identify $C_{11}=2 U_{\mathrm{RVE}} / V_{\mu}$.

(2) Uniaxial extension for $C_{22}$ : we apply a uniform strain $\varepsilon_{y y}=1$ and shear stress $\sigma_{x y}=\sigma_{y z}=\sigma_{x z}=0$ on the unit cell's boundary, the corresponding boundary conditions are then written as

$$
\begin{aligned}
& u_{x}=0, \sigma_{x y} n_{1}=0, \sigma_{x z} n_{1}=0 \text { on } \mathrm{n}_{1} \text {-face, } \\
& u_{y}=y, \sigma_{x y} n_{2}=0, \sigma_{y z} n_{2}=0 \text { on } \mathrm{n}_{2} \text {-face, } \\
& u_{z}=0, \sigma_{x z} n_{3}=0, \sigma_{y z} n_{3}=0 \text { on } \mathrm{n}_{3} \text {-face, }
\end{aligned}
$$

which gives $C_{22}=2 U_{\mathrm{RVE}} / V_{\mu}$.

(3) Uniaxial extension for $C_{33}$ : we apply a uniform strain $\varepsilon_{z z}=1$ and shear stress $\sigma_{x y}=\sigma_{y z}=\sigma_{x z}=0$ on the unit cell's boundary, the corresponding boundary conditions are then written as

$$
\begin{aligned}
& u_{x}=0, \sigma_{x y} n_{1}=0, \sigma_{x z} n_{1}=0 \text { on } \mathrm{n}_{1} \text {-face, } \\
& u_{y}=0, \sigma_{x y} n_{2}=0, \sigma_{y z} n_{2}=0 \text { on } \mathrm{n}_{2} \text {-face, } \\
& u_{z}=z, \sigma_{x z} n_{3}=0, \sigma_{y z} n_{3}=0 \text { on } \mathrm{n}_{3} \text {-face, }
\end{aligned}
$$

this gives $C_{33}=2 U_{\mathrm{RVE}} / V_{\mu}$.

(4) Biaxial extension for $C_{12}$ : we apply a uniform strain $\varepsilon_{x x}=\varepsilon_{y y}=1$ and shear stress $\sigma_{x y}=\sigma_{y z}=\sigma_{x z}=0$ on the unit cell's boundary, the corresponding mixed boundary conditions are then written as

$$
\begin{array}{lll}
u_{x}=x, \sigma_{x y} n_{1}=0, \sigma_{x z} n_{1}=0 & \text { on } & \mathrm{n}_{1} \text {-face, } \\
u_{y}=y, \sigma_{x y} n_{2}=0, \sigma_{y z} n_{2}=0 & \text { on } & \mathrm{n}_{2} \text {-face, } \\
u_{z}=0, \sigma_{x z} n_{3}=0, \sigma_{y z} n_{3}=0 & \text { on } & \mathrm{n}_{3} \text {-face, }
\end{array}
$$

which yields $C_{12}=\left(2 U_{\mathrm{RVE}} / V_{\mu}-C_{11}-C_{22}\right) / 2$.

(5) Biaxial extension for $C_{23}$ : we apply a uniform strain $\varepsilon_{y y}=\varepsilon_{z z}=1$ and shear stress $\sigma_{x y}=\sigma_{y z}=\sigma_{x z}=0$ on the unit cell's boundary, the corresponding boundary conditions are then written as

$$
\begin{aligned}
& u_{x}=0, \sigma_{x y} n_{1}=0, \sigma_{x z} n_{1}=0 \text { on } \mathrm{n}_{1} \text {-face, } \\
& u_{y}=y, \sigma_{x y} n_{2}=0, \sigma_{y z} n_{2}=0 \text { on } \mathrm{n}_{2} \text {-face, } \\
& u_{z}=z, \sigma_{x z} n_{3}=0, \sigma_{y z} n_{3}=0 \text { on } \mathrm{n}_{3} \text {-face, }
\end{aligned}
$$

which yields $C_{23}=\left(2 U_{\mathrm{RVE}} / V_{\mu}-C_{22}-C_{33}\right) / 2$.

(6) Biaxial extension for $C_{13}$ : we apply a uniform strain $\varepsilon_{x x}=\varepsilon_{z z}=1$ and shear stress $\sigma_{x y}=\sigma_{y z}=\sigma_{x z}=0$ on the unit cell's boundary, the corresponding boundary conditions are then written as

$$
\begin{array}{lll}
u_{x}=x, \sigma_{x y} n_{1}=0, \sigma_{x z} n_{1}=0 & \text { on } & \mathrm{n}_{1} \text {-face, } \\
u_{y}=0, \sigma_{x y} n_{2}=0, \sigma_{y z} n_{2}=0 & \text { on } & \mathrm{n}_{2} \text {-face, } \\
u_{z}=z, \sigma_{x z} n_{3}=0, \sigma_{y z} n_{3}=0 & \text { on } & \mathrm{n}_{3} \text {-face, }
\end{array}
$$

which yields $C_{13}=\left(2 U_{\mathrm{RVE}} / V_{\mu}-C_{11}-C_{33}\right) / 2$. 
T A B L E 1 Calculated moduli $C_{i j}$ in MPa for the simulated auxetic samples

\begin{tabular}{|c|c|c|c|c|c|c|c|c|c|}
\hline \multirow[b]{2}{*}{ Cell sizes } & \multicolumn{3}{|c|}{ Uniaxial tensile tests } & \multicolumn{3}{|c|}{ Biaxial tensile tests } & \multicolumn{3}{|c|}{ Shear tests } \\
\hline & $\overline{C_{11}}$ & $C_{22}$ & $C_{33}$ & $\overline{C_{12}}$ & $C_{23}$ & $C_{13}$ & $\overline{C_{44}}$ & $C_{55}$ & $C_{66}$ \\
\hline $1 \times 1$ & 93.9 & 92.4 & 821.8 & -78.3 & 4.97 & 5.45 & 3.21 & 76.7 & 78.0 \\
\hline $4 \times 4$ & 95.5 & 93.9 & 820.0 & -80.0 & 4.98 & 5.58 & 3.27 & 76.5 & 78.0 \\
\hline $8 \times 8$ & 102.6 & 100.6 & 818.6 & -85.0 & 5.40 & 6.00 & 3.32 & 77.8 & 79.0 \\
\hline
\end{tabular}

(7) Shear deformation for $C_{44}$ : we apply a uniform shear strain $\varepsilon_{x y}=1$ and normal stress $\sigma_{x x}=\sigma_{y y}=\sigma_{z z}=0$ on the unit cell's boundary, the corresponding boundary conditions are then written as

$$
\begin{aligned}
& u_{x}=y / 2, \sigma_{x y} n_{1}=0, \sigma_{x z} n_{1}=0 \quad \text { on } \mathrm{n}_{1} \text {-face, } \\
& u_{y}=x / 2, \sigma_{x y} n_{2}=0, \sigma_{y z} n_{2}=0 \quad \text { on } \mathrm{n}_{2} \text {-face, } \\
& u_{z}=0, \quad \sigma_{x z} n_{3}=0, \sigma_{y z} n_{3}=0 \quad \text { on } \quad \mathrm{n}_{3} \text {-face, }
\end{aligned}
$$

which yields $C_{44}=2 U_{\mathrm{RVE}} / V_{\mu}$.

(8) Shear deformation for $C_{55}$ : we apply a uniform shear strain $\varepsilon_{y z}=1$ and normal stress $\sigma_{x x}=\sigma_{y y}=\sigma_{z z}=0$ on the unit cell's boundary; the corresponding boundary conditions are then written as

$$
\begin{aligned}
& u_{x}=0, \quad \sigma_{x y} n_{1}=0, \sigma_{x z} n_{1}=0 \quad \text { on } \mathrm{n}_{1} \text {-face, } \\
& u_{y}=z / 2, \sigma_{x y} n_{2}=0, \sigma_{y z} n_{2}=0 \quad \text { on } \mathrm{n}_{2} \text {-face, } \\
& u_{z}=y / 2, \sigma_{x z} n_{3}=0, \sigma_{y z} n_{3}=0 \quad \text { on } \mathrm{n}_{3} \text {-face, }
\end{aligned}
$$

which yields $C_{55}=2 U_{\mathrm{RVE}} / V_{\mu}$.

(9) Shear deformation for $C_{66}$ : we apply a uniform shear strain $\varepsilon_{x z}=1$ and normal stress $\sigma_{x x}=\sigma_{y y}=\sigma_{z z}=0$ on the unit cell's boundary, the corresponding boundary conditions are then written as

$$
\begin{aligned}
& u_{x}=z / 2, \sigma_{x y} n_{1}=0, \sigma_{x z} n_{1}=0 \quad \text { on } \mathrm{n}_{1} \text {-face, } \\
& u_{y}=0, \quad \sigma_{x y} n_{2}=0, \sigma_{y z} n_{2}=0 \quad \text { on } \mathrm{n}_{2} \text {-face, } \\
& u_{z}=x / 2, \sigma_{x z} n_{3}=0, \sigma_{y z} n_{3}=0 \quad \text { on } \quad \mathrm{n}_{3} \text {-face, }
\end{aligned}
$$

which yields $C_{66}=2 U_{\mathrm{RVE}} / V_{\mu}$.

The classical and non-classical bending coefficients are derived computationally using the FE method. The auxetic unit cells have been meshed by a combination of linear hexahedral elements C3D8R and linear wedge elements C3D6 used in ABAQUS environment with an approximate global size of $0.2 \mathrm{~mm}$. The computed $C_{i j}$ coefficients are given in Table 1 . One can conclude from the results that mixed displacement and traction boundary conditions give effective elastic coefficients $C_{i j}$ which are relatively independent of unit cell sizes.

We observe that the structure is as expected nearly in-plane orthotropic, since the two in-plane tensile moduli are very close to each other. From the results, it appears that the rigidity coefficients $C_{11}$ and $C_{33}$ are different: this is expected, since $C_{11}$ is computed in x-direction, whereas $C_{33}$ is evaluated in the z-direction which is stiffer. This difference in the rigidity values also results from the kinematics applied on the boundaries of the representative volume element to evaluate these two moduli, which are quite different, as previously shown. The constitutive law relating the equivalent stress components to the strain components, $\operatorname{viz}\{\sigma\}=[C]\{\varepsilon\}$, can then be written as 
T A B L E 2 Calculated engineering constants in MPa for the simulated auxetic samples

\begin{tabular}{|c|c|c|c|c|c|c|}
\hline Cell sizes & $E_{x}$ & $E_{y}$ & $E_{z}$ & $G_{x y}$ & $G_{y z}$ & $\boldsymbol{G}_{x z}$ \\
\hline $1 \times 1$ & 27.5 & 27.0 & 818 & 3.21 & 76.7 & 78.0 \\
\hline $2 \times 2$ & 27.0 & 26.5 & 816 & 3.25 & 76.4 & 77.8 \\
\hline $8 \times 8$ & 30.6 & 30.0 & 815 & 3.32 & 77.8 & 79.0 \\
\hline
\end{tabular}

T A B L E 3 Calculated in-plane and out-of-plane Poisson's ratio for the simulated auxetic samples

\begin{tabular}{lllllll} 
Cell sizes & $\boldsymbol{v}_{x y}$ & $\boldsymbol{v}_{\boldsymbol{y}}$ & $\boldsymbol{v}_{\boldsymbol{z}}$ & $\boldsymbol{v}_{z y}$ & $\boldsymbol{v}_{x z}$ & 0.0117 \\
$1 \times 1$ & -0.850 & -0.834 & 0.0115 & 0.350 & 0.349 \\
$2 \times 2$ & -0.854 & -0.838 & 0.0116 & 0.359 & 0.0119 & 0.359 \\
$4 \times 4$ & -0.851 & -0.839 & 0.0117 & 0.359 & 0.0119 & 0.359 \\
$8 \times 8$ & -0.844 & -0.827 & 0.0126 & 0.343 & 0.0128 & 0.343 \\
\hline
\end{tabular}

$$
\left\{\begin{array}{c}
\sigma_{x x} \\
\sigma_{y y} \\
\sigma_{z z} \\
\sigma_{x y} \\
\sigma_{y z} \\
\sigma_{x z}
\end{array}\right\}=\left[\begin{array}{cccccc}
C_{11} & C_{12} & C_{13} & 0 & 0 & 0 \\
C_{12} & C_{22} & C_{23} & 0 & 0 & 0 \\
C_{13} & C_{23} & C_{33} & 0 & 0 & 0 \\
0 & 0 & 0 & C_{44} & 0 & 0 \\
0 & 0 & 0 & 0 & C_{55} & 0 \\
0 & 0 & 0 & 0 & 0 & C_{66}
\end{array}\right]\left\{\begin{array}{c}
\varepsilon_{x x} \\
\varepsilon_{y y} \\
\varepsilon_{z z} \\
\varepsilon_{x y} \\
\varepsilon_{y z} \\
\varepsilon_{x z}
\end{array}\right\}
$$

Therefore, one derives the effective traction moduli from the effective compliance matrix $[S]=[C]^{-1}$, as

$$
E_{x}=\left[1 / S_{11}\right], E_{y}=\left[1 / S_{22}\right], E_{z}=\left[1 / S_{33}\right] \text {. }
$$

Furthermore, the effective in-plane Poisson's ratios are computed from the relations

$$
v_{x y}=-S_{21} E_{x} v_{y x}=-S_{12} E_{y}
$$

and the out-of-plane Poisson's ratios are similarly computed from the relations

$$
v_{y z}=-S_{32} E_{y}, v_{z y}=-S_{23} E_{z}, v_{x z}=-S_{31} E_{x}, v_{z x}=-S_{13} E_{z}
$$

The effective shear moduli are additionally computed respectively as

$$
G_{x y}=\left[1 / S_{44}\right], G_{y z}=\left[1 / S_{55}\right], G_{x z}=\left[1 / S_{66}\right] \text {. }
$$

The above mentioned Young's and shear moduli as well as Poisson's ratio are calculated from the coefficients $C_{i j}$ in Table 1 for different auxetic unit cells and the results are displayed in Tables 2 and 3. The computed in plane Poisson's ratio ( -0.84 ) is very close to the measured ones $(-0.8$, representing the average value over the different tests) and to the optimal Poisson'a ratio $(-0.82)$ obtained from topology optimization, the differences originating from the uncertainty in the measured elastic properties of the bulk material, and the imperfect representation of the sample geometry. The effective relative density of the samples has been measured by image analysis (counting the relative amount of black and white pixels, respectively corresponding to the presence and absence of material) as 0.4 ; in comparison, the computed effective relative density is 0.41 , so it is very close to the measured one.

The displacement distributions within $2 \times 2$ unit cells of auxetic structure due to uniaxial strain loadings $\varepsilon_{x x}=1, \varepsilon_{z z}=1$, biaxial strain loadings

$$
\varepsilon_{x x}=\varepsilon_{y y}=1, \varepsilon_{x x}=\varepsilon_{z z}=1
$$

and shear loading $\varepsilon_{x y}=1$ are exposed in Figures 11-15.

The displacement distributions within $8 \times 8$ unit cells of auxetic structure due to uniaxial strain loading $\varepsilon_{x x}=1$, biaxial strain loading $\varepsilon_{x x}=\varepsilon_{y y}=1$, and shear loading $\varepsilon_{x y}=1$, are additionally exposed in Figures 16-18. 


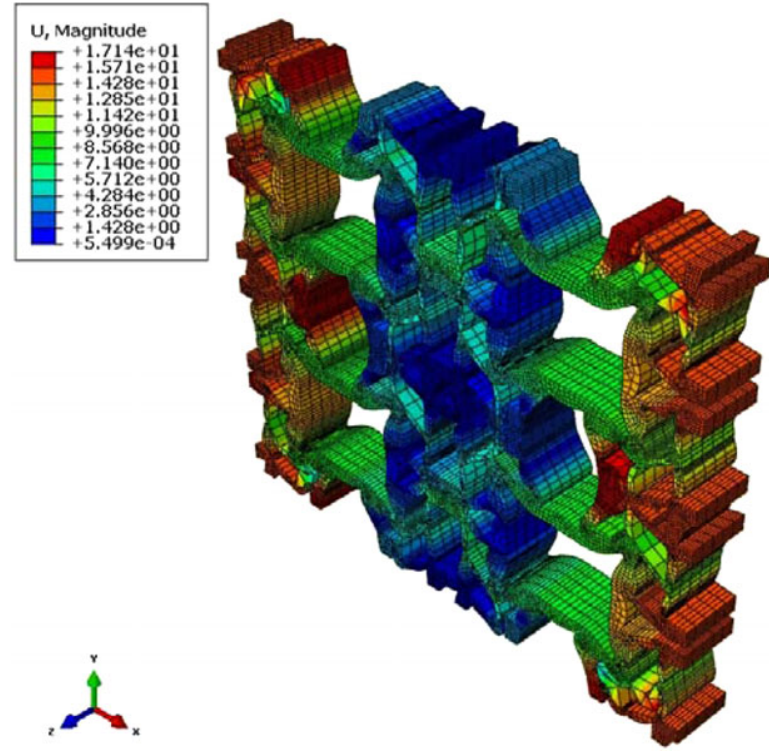

F I G U R E 11 Displacement distributions within $2 \times 2$ unit cells of the auxetic structure due to uniaxial extension along $x\left(\varepsilon_{x x}=1\right)$

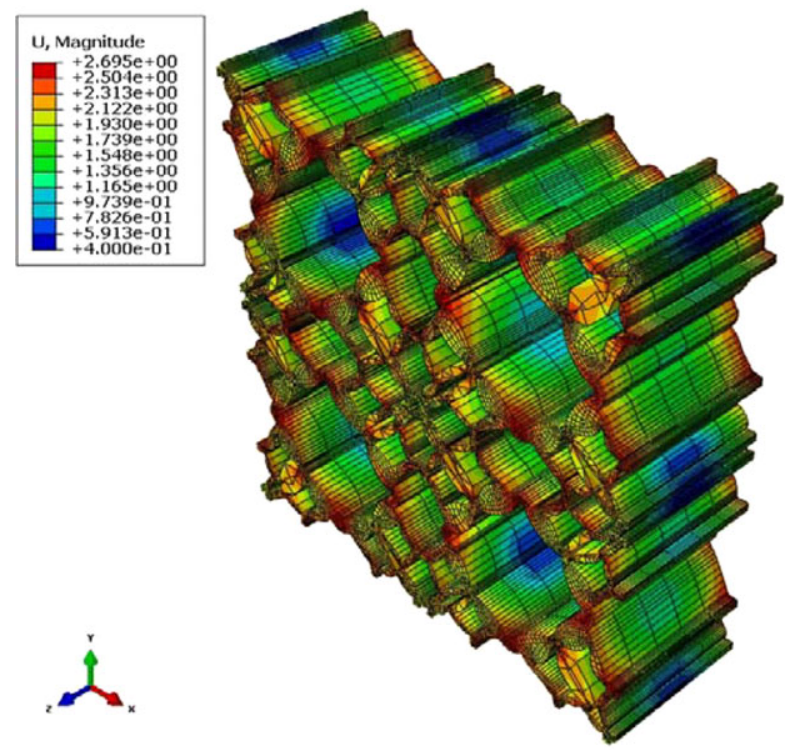

F I G URE 12 Displacement distributions within $2 \times 2$ unit cells of the auxetic structure due to uniaxial extension along $z\left(\varepsilon_{z z}=1\right)$

We extract from the effective compliance matrix an effective Poisson's ratio equal to -0.84 (we consider the value obtained for the largest tested RVE size). An additional set of twelve tests is actually required to identify the higher order moduli associated with the couple-stress theory; these tests are described in the sequel.

(1) Uniaxial torsional rotation for $D_{11}$ : we apply a uniform torsional rotation $\chi_{x x}=1$ and specify zero shear tractions on the unit cell's boundary, thus the corresponding boundary conditions are written as

$$
u_{x}=0, u_{y}=-x z, u_{z}=x y \text { on } \mathrm{n}_{1} \text {-face, }
$$

which gives $D_{11}=2 U_{\mathrm{RVE}} / V_{\mu}$.

(2) Uniaxial torsional rotation for $D_{22}$ : we apply a uniform torsional rotation $\chi_{y y}=1$ and specify zero shear tractions on the unit cell's boundary, thus the corresponding boundary conditions are written as

$$
u_{x}=y z, u_{y}=0, u_{z}=-x y \text { on } \mathrm{n}_{2} \text {-face, }
$$

which gives $D_{22}=2 U_{\mathrm{RVE}} / V_{\mu}$. 

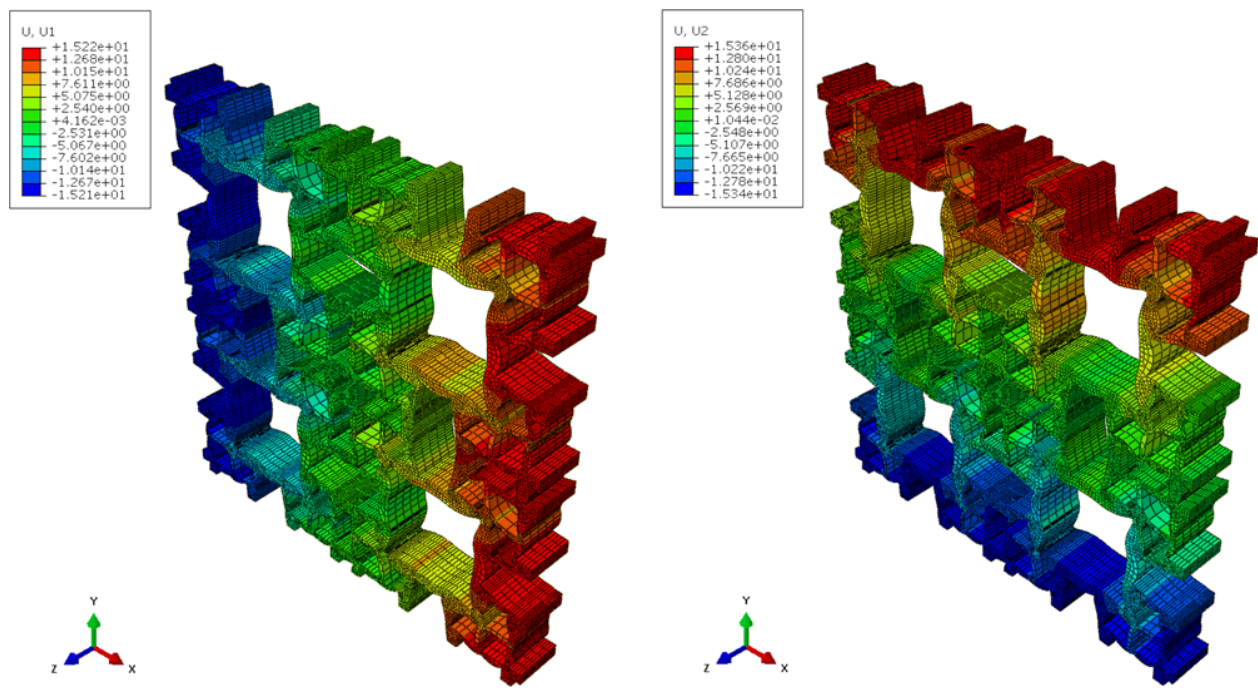

F I G U RE 13 Displacement distributions within $2 \times 2$ unit cells of the auxetic structure due to biaxial extension $\varepsilon_{x x}=\varepsilon_{y y}=1$
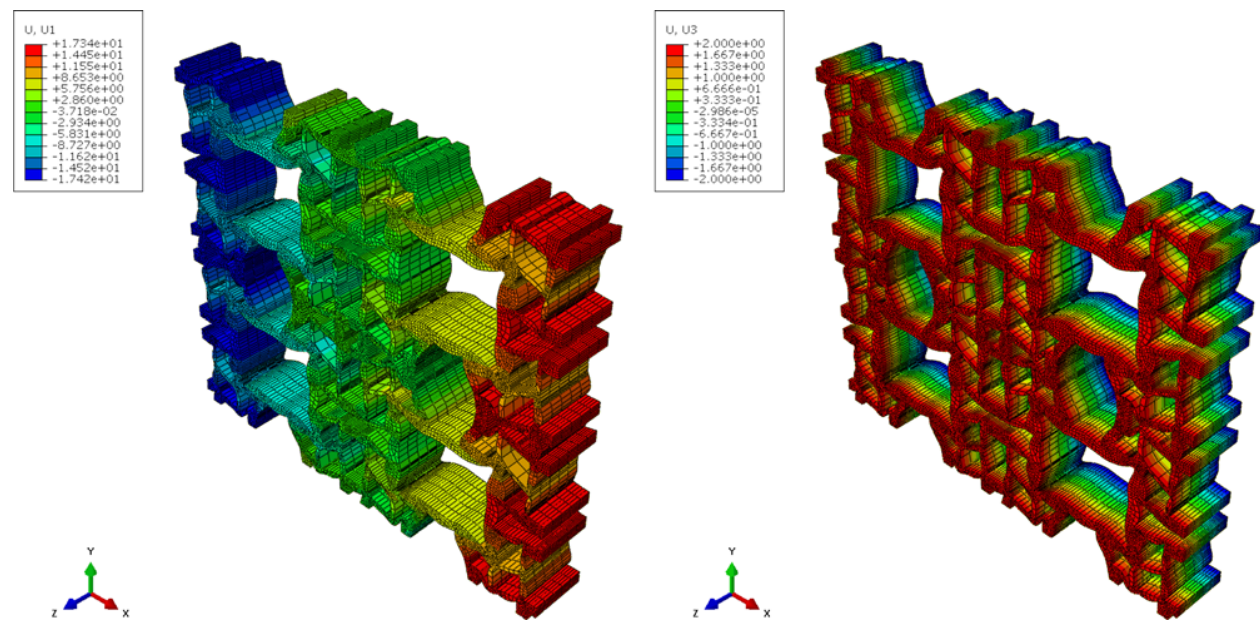

F I G U R E 14 Displacement distributions within $2 \times 2$ unit cells of the auxetic structure due to biaxial extension $\varepsilon_{x x}=\varepsilon_{z z}=1$

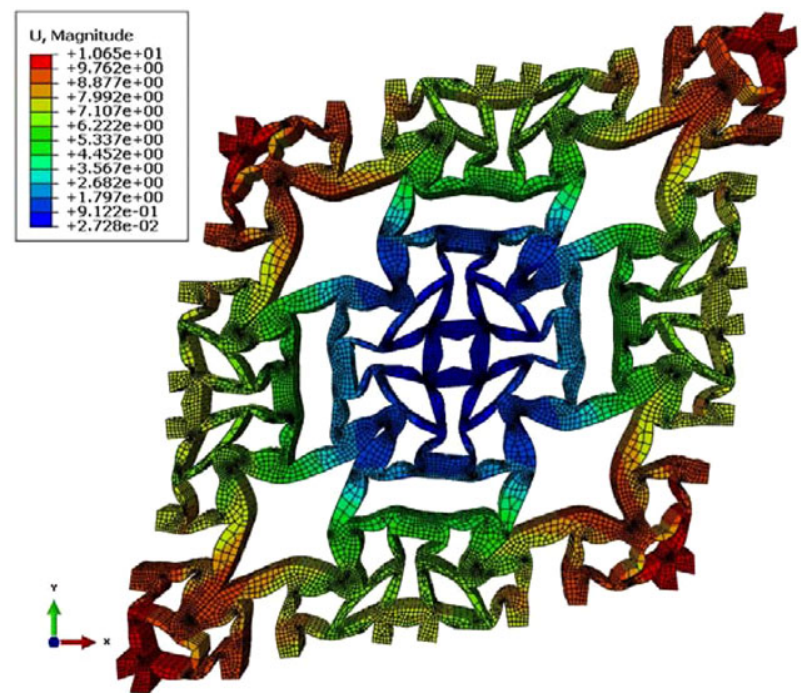

F I G U R E 15 Displacement distributions within $2 \times 2$ unit cells of the auxetic structure due to shear loading $\varepsilon_{x y}=1$ 


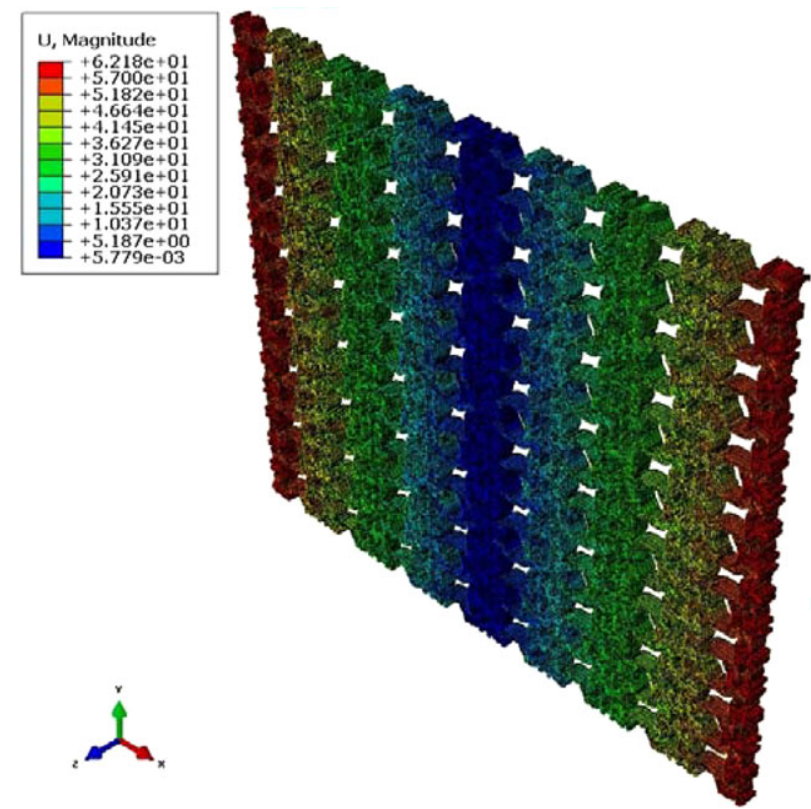

F I G U R E 16 Displacement distributions within $8 \times 8$ unit cells of auxetic structure due to uniaxial extension along $x\left(\varepsilon_{x x}=1\right)$
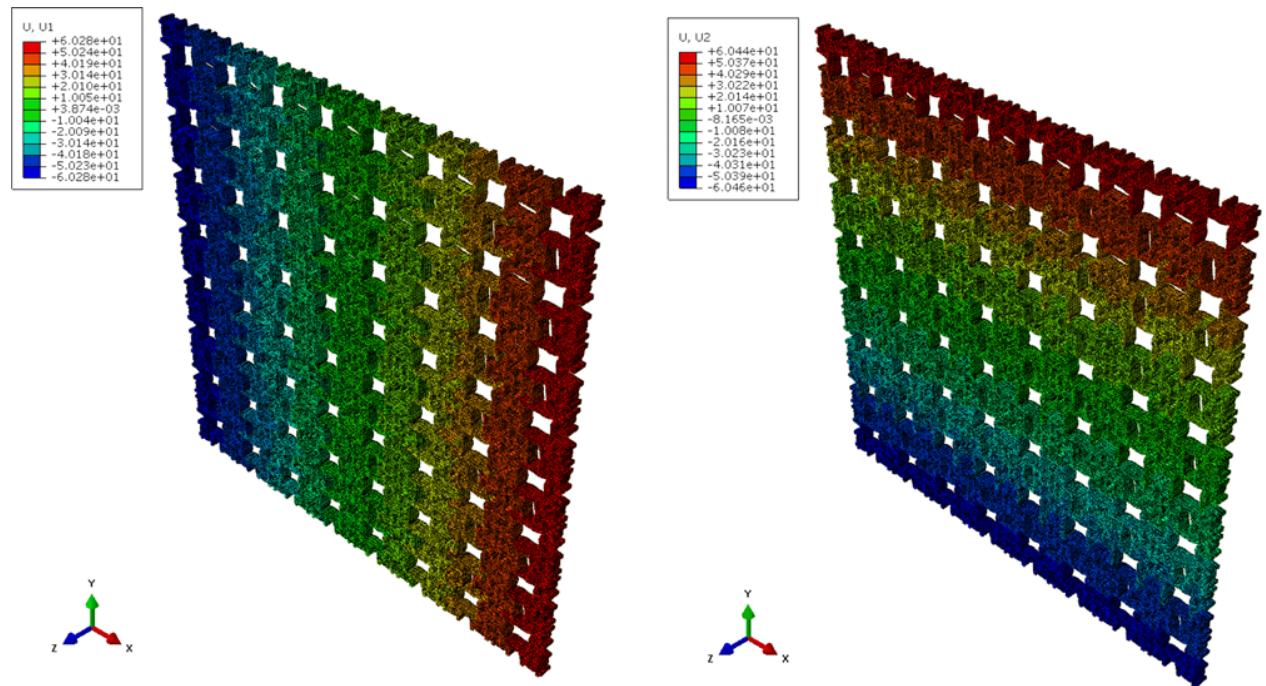

F I G URE 17 Displacement distributions within $8 \times 8$ unit cells of auxetic structure due to biaxial extension $\varepsilon_{x x}=\varepsilon_{y y}=1$

(3) Uniaxial torsional rotation for $D_{33}$ : we apply a uniform torsional rotation $\chi_{z z}=1$ and specify zero shear tractions on the unit cell's boundary, thus the corresponding boundary conditions are written as

$$
u_{x}=-y z, u_{y}=x z, u_{z}=0 \text { on } \mathrm{n}_{3} \text {-face, }
$$

which gives $D_{33}=2 U_{\mathrm{RVE}} / V_{\mu}$.

(4) Biaxial torsional rotation for $D_{12}$ : we apply a uniform torsional rotation $\chi_{x x}=\chi_{y y}=1$ and specify zero shear tractions on the unit cell's boundary, thus the corresponding boundary conditions are written as

$$
\begin{aligned}
& u_{y}=-x z, u_{z}=x y \text { on } \mathrm{n}_{1} \text {-face, } \\
& u_{x}=y z, u_{z}=-x y \text { on } \mathrm{n}_{2} \text {-face, }
\end{aligned}
$$

which gives $D_{12}=\left(2 U_{\mathrm{RVE}} / V_{\mu}-D_{11}-D_{22}\right) / 2$. 


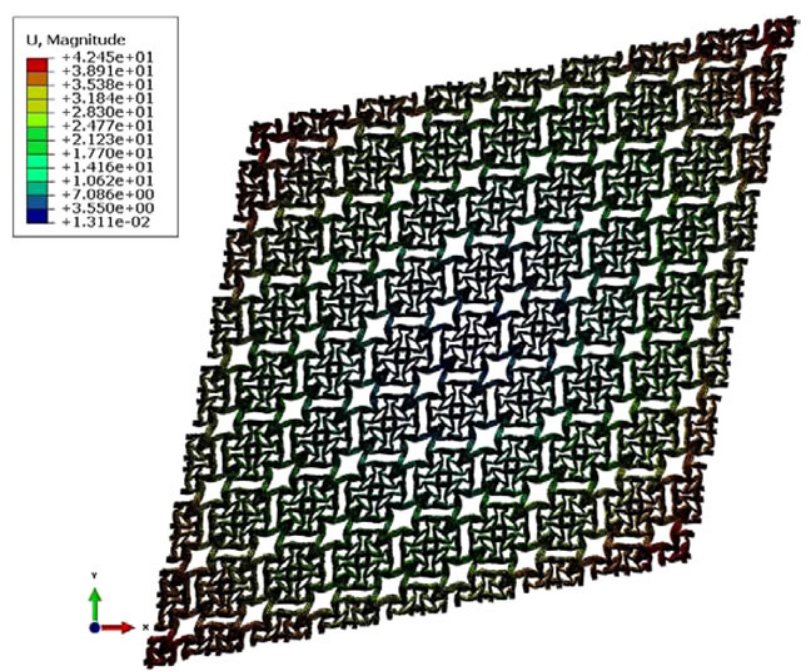

F I G U R E 18 Displacement distributions within $8 \times 8$ unit cells of auxetic structure due to shear strain $\varepsilon_{x y}=1$

(5) Biaxial torsional rotation for $D_{23}$ : when we apply a uniform torsional rotation $\chi_{y y}=\chi_{z z}=1$ on the unit cell's boundary, the corresponding boundary conditions are then written as

$$
\begin{aligned}
& u_{x}=y z, u_{z}=-x y \text { on } \mathrm{n}_{2} \text {-face, } \\
& u_{x}=-y z, u_{y}=x z \text { on } \mathrm{n}_{3} \text {-face, }
\end{aligned}
$$

which yields $D_{23}=\left(2 U_{\mathrm{RVE}} / V_{\mu}-D_{22}-D_{33}\right) / 2$.

(6) Biaxial torsional rotation for $D_{13}$ : when we apply a uniform torsional rotation $\chi_{x x}=\chi_{z z}=1$ on the unit cell's boundary, the corresponding boundary conditions are then written as

$$
\begin{aligned}
& u_{y}=-x z, u_{z}=x y \text { on } \mathrm{n}_{1} \text {-face, } \\
& u_{x}=-y z, u_{y}=x z \text { on } \mathrm{n}_{3} \text {-face, }
\end{aligned}
$$

which yields $D_{13}=\left(2 U_{\mathrm{RVE}} / V_{\mu}-D_{11}-D_{33}\right) / 2$.

(7) Uniform curvature for $D_{44}$ : when we apply a uniform curvature $\chi_{x y}=1$ on the unit cell's boundary, the corresponding boundary conditions are then given as

$$
u_{x}=x z \text { on } \mathrm{n}_{1} \text {-face, } u_{y}=0 \text { on } \mathrm{n}_{2} \text {-face, } u_{z}=-x^{2} / 2 \text { on } \mathrm{n}_{3} \text {-face, }
$$

which yields $D_{44}=2 U_{\mathrm{RVE}} / V_{\mu}-C_{11} z^{2}$.

(8) Uniform curvature for $D_{55}$ : when we apply a uniform curvature $\chi_{y x}=1$ on the unit cell's boundary, the corresponding boundary conditions are then given as

$$
u_{x}=0 \text { on } \mathrm{n}_{1} \text {-face, } u_{y}=-y z \text { on } \mathrm{n}_{2} \text {-face, } u_{z}=y^{2} / 2 \text { on } \mathrm{n}_{3} \text {-face, }
$$

which yields $D_{55}=2 U_{\mathrm{RVE}} / V_{\mu}-C_{22} z^{2}$.

(9) Uniform curvature for $D_{66}$ : when we apply a uniform curvature $\chi_{y z}=1$ on the unit cell's boundary, the corresponding boundary conditions are then given as

$$
u_{x}=-y^{2} / 2 \text { on } \mathrm{n}_{1} \text {-face, } u_{y}=x y \text { on } \mathrm{n}_{2} \text {-face, } u_{z}=0 \text { on } \mathrm{n}_{3} \text {-face, }
$$

which gives $D_{66}=2 U_{\mathrm{RVE}} / V_{\mu}-C_{22} x^{2}$. 
TA B L E 4 Calculated bending curvature moduli $D_{i j}$ in $\mathrm{N}$ for the simulated auxetic samples

\begin{tabular}{|c|c|c|c|c|}
\hline \multirow[b]{2}{*}{ Cells size } & \multicolumn{4}{|c|}{ Bending curvature moduli } \\
\hline & $\overline{D_{44}}$ & $D_{55}$ & $D_{66}$ & $D_{88}$ \\
\hline $1 \times 1$ & 268 & 266 & 419 & 448 \\
\hline $4 \times 4$ & 269 & 267 & 7804 & 7997 \\
\hline $8 \times 8$ & 271 & 269 & 35308 & 35986 \\
\hline
\end{tabular}

(10) Uniform curvature for $D_{77}$ : when we apply a uniform curvature $\chi_{z y}=1$ on the unit cell's boundary, the corresponding boundary conditions are then given as

$$
u_{x}=-x y \text { on } \mathrm{n}_{1} \text {-face, } u_{y}=x^{2} / 2 \text { on } \mathrm{n}_{2} \text {-face, } u_{z}=0 \text { on } \mathrm{n}_{3} \text {-face, }
$$

which yields $D_{77}=2 U_{\mathrm{RVE}} / V_{\mu}-C_{33} x^{2}$.

(11) Uniform curvature for $D_{88}$ : when we apply a uniform bending curvature $\chi_{x z}=1$ on the unit cell's boundary, the corresponding boundary conditions are then written as

$$
u_{x}=-x y \text { on } \mathrm{n}_{1} \text {-face, } u_{y}=x^{2} / 2 \text { on } \mathrm{n}_{2} \text {-face, } u_{z}=0 \text { on } \mathrm{n}_{3} \text {-face, }
$$

which yields $D_{88}=2 U_{\mathrm{RVE}} / V_{\mu}-C_{11} y^{2}$.

(12) Uniform curvature for $D_{99}$ : When we apply a uniform curvature $\chi_{z x}=1$ on the unit cell's boundary, the corresponding boundary conditions are then given as

$$
u_{x}=0 \text { on } \mathrm{n}_{1} \text {-face, } u_{y}=-z^{2} / 2 \text { on } \mathrm{n}_{2} \text {-face, } u_{z}=y z \text { on } \mathrm{n}_{2} \text {-face, }
$$

which yields $D_{99}=2 U_{\mathrm{RVE}} / V_{\mu}-C_{33} y^{2}$.

However we presently focus on the interesting bending curvatures behaviors for those auxetic lattices.

The derived couple-stress moduli do in fact not depend on the choice the origin of the coordinate system with respect to the centroid of the tested sample. The additional terms involving the coordinate vanish when the origin of the coordinate axes is selected as the centroid of the tested sample. The calculated bending curvature components of stiffness tensor $D_{i j}$ of the auxetic samples with different sizes are reported in Table 4. The dependency of the in-plane bending moduli $D_{66}$ and $D_{88}$ on the size of the auxetic structures is very clear, due to the fact that these moduli increase with the auxetic cell size. On the other hand the out-of-plane bending moduli $D_{44}$ and $D_{55}$ appear to be independent of the cell size, thus they can be considerdd as intrinsic to the microstructure itself. Observe from previous Tables that the microstructure is symmetrical but in fact not isotropic, since the classical tensile moduli in x and y directions are slightly different; similarly, the bending moduli $D_{66}$ and $D_{88}$ also differ as a consequence of the lack of isotropy.

The iso-displacements within $2 \times 2$ and $8 \times 8$ unit cells of auxetic samples due to out-of-plane bending curvatures $\chi_{x y}=1$ and in-plane bending curvatures $\chi_{y z}=1$ and $\chi_{x z}=1$ are respectively pictured in Figures 19, 20, and 21.

The same computations and plots are repeated for the set of $8 \times 8$ unit cells of the auxetic structure, as illustrated in Figures 22, 23 , and 24.

A further justification of the choice of the couple stress model can be found considering the macroscale behavior. Based on the previous auxetic homogenized properties (e.g., a $1 \times 1$ cell), we will quantify the effects of the couple stress model on the macroscopic scale by evaluating the response of a macrobeam subjected to a pure bending moment: we shall compare the simplified equation of a micropolar beam incorporating auxetic microstructures under pure bending to the standard bending equations of classical beams. The geometrical parameters of the macrobeam are indicated in Figure 25: $\mathrm{H}$ and $\mathrm{L}$ are the beam height and length, respectively. The macrobeam incorporates the optimal microstructure determined in previous sections by topology optimization, and which is repeated along the beam axis by periodic translation. 


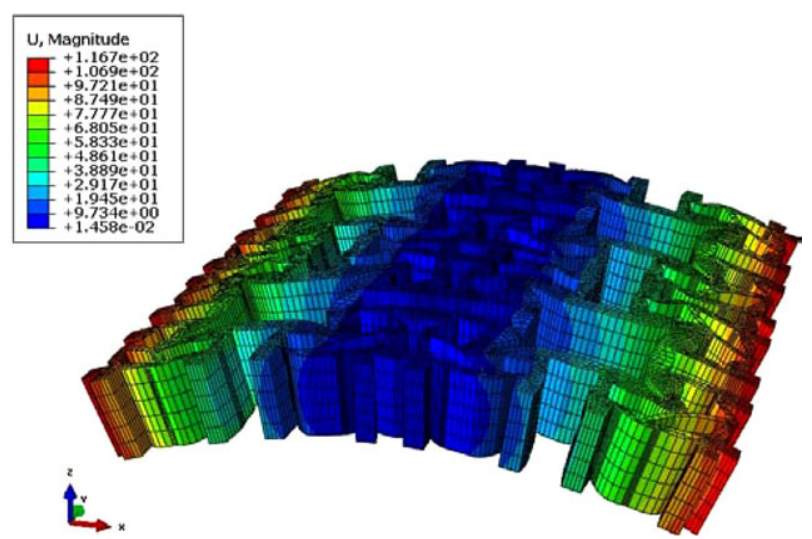

F I G U R E 19 Displacement distributions over $2 \times 2$ unit cells of auxetic structure due to out-of-plane bending curvature $\chi_{x y}=1$

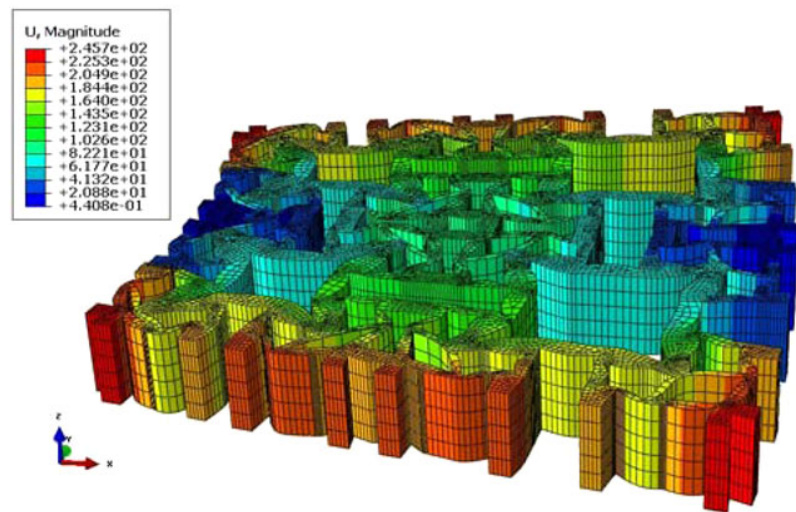

F I G U R E 20 Displacement distributions over $2 \times 2$ unit cells of auxetic structure due to in-plane bending curvature $\chi_{y z}=1$

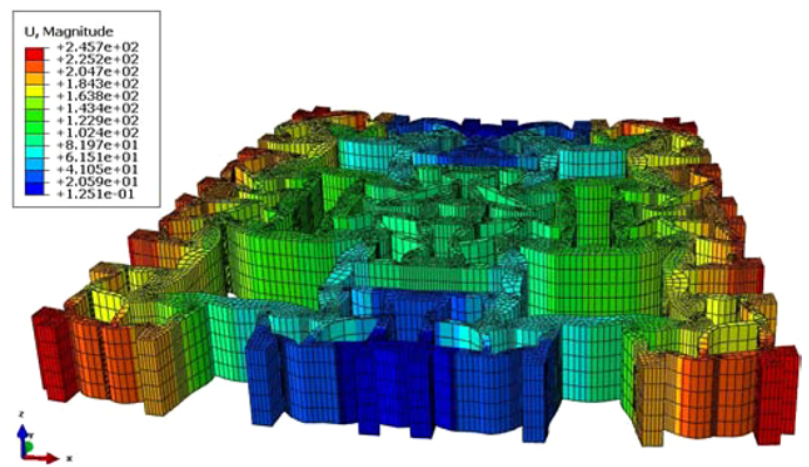

F I G U R E 21 Displacement distributions over $2 \times 2$ unit cells of auxetic structure due to in-plane bending curvature $\chi_{x z}=1$

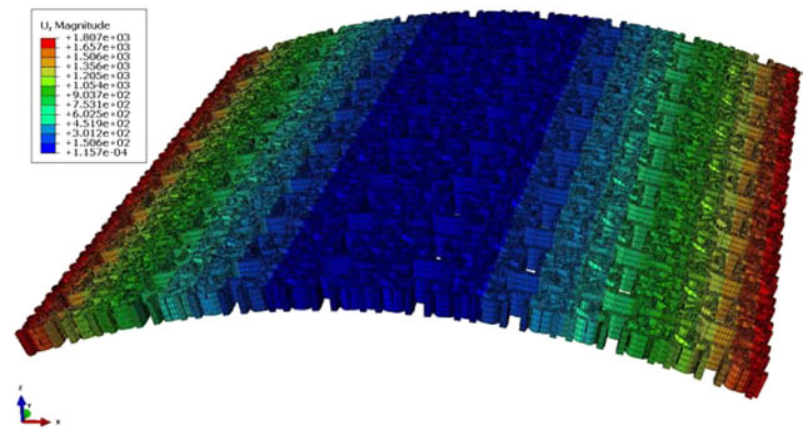

F I G U R E 22 Displacement distributions over $8 \times 8$ unit cells of auxetic structure due to out-of-plane bending curvature $\chi_{x y}=1$ 


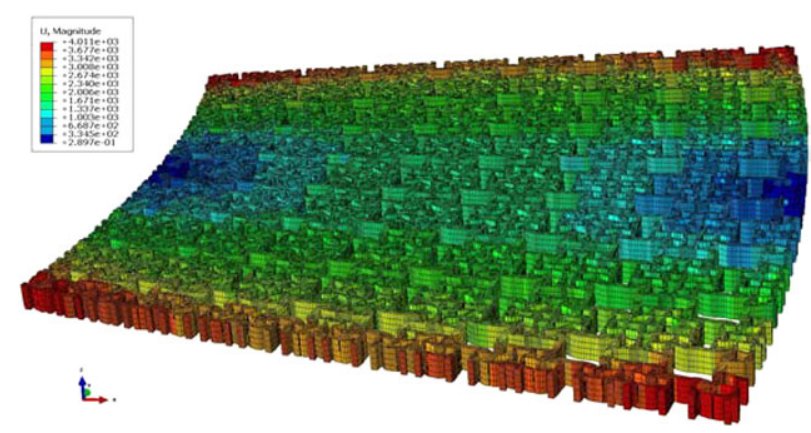

F I G URE 23 Displacement distributions over $8 \times 8$ unit cells of auxetic structure due to in-plane bending curvature $\chi_{y z}=1$

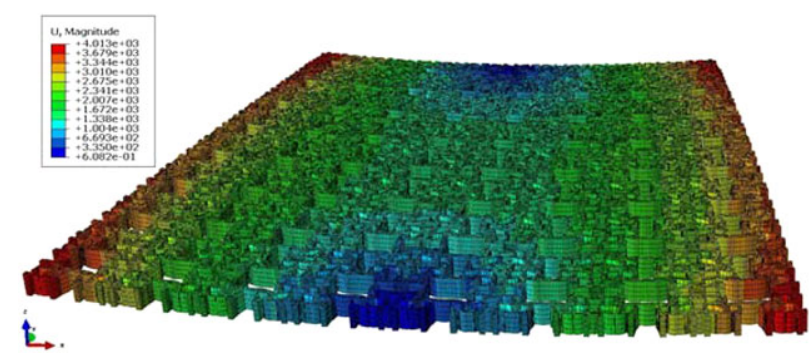

F I G U R E 24 Displacement distributions over $8 \times 8$ unit cells of auxetic structure due to in-plane bending curvature $\chi_{x z}=1$

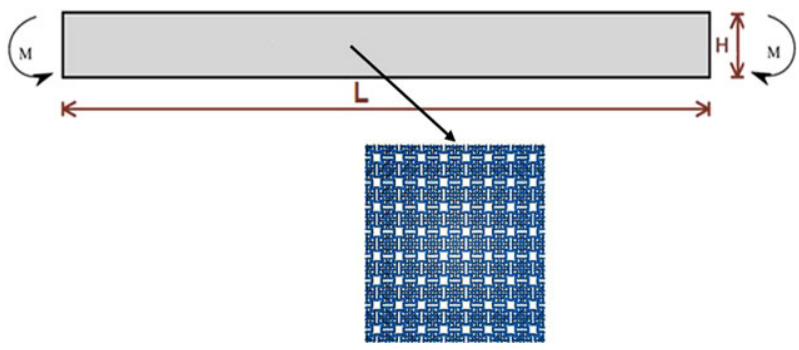

F I G U R E 25 Elastic beam with auxetic microstructures subjected to pure bending

The equilibrium equations based on couple-stress elasticity here of an elastic beam subjected to a uniform bending moment $\mathrm{M}$ are given by

$$
\underbrace{\left(E_{x} I_{z}+D_{88} H\right)}_{\kappa_{\text {couple-stress }}^{x z}} \frac{\partial \phi_{z}}{\partial x}=-M_{x} ; \underbrace{\left(E_{y} I_{z}+D_{66} H\right)}_{\kappa_{\text {couple-stress }}^{y z}} \frac{\partial \phi_{z}}{\partial y}=-M_{y}
$$

these equations represent successively bending in the $x-z$ and $y-z$ planes, with $E_{x}$ and $E_{y}$ therein the effective homogenized moduli of the macroscopic equivalent beam, $\mathrm{M}$ the bending moment, $I_{z}$ the quadratic moment of the macroscopic beam along $\mathrm{z}$, and $\kappa_{\text {couple-stress }}^{x z}, \kappa_{\text {couple-stress }}^{y z}$ are the bending stiffnesses of the beam (exhibiting couplestress effect). The parameter $H$ is the height of the auxetic structure. The characteristic length that intervenes in previous beam bending equation is an essential parameter in the couple-stress model, thus we write the definition of this parameter for an orthotropic continuum in terms of the engineering constants. The characteristic lengths can be extracted from the micropolar constitutive law, corresponding to the bending couple-stress components; They can be computed as follows: ${ }^{[9,10,14]}$

$$
l_{b 1}=\sqrt{\frac{D_{88}}{4 C_{44}}}, l_{b 2}=\sqrt{\frac{D_{66}}{4 C_{44}}} .
$$


The previous bending equations can then be written in terms of the characteristic bending length $l_{b}$ as:

$$
\underbrace{\left(E_{x} I_{z}+4 l_{b 1}^{2} C_{44} H\right)}_{\kappa_{\text {couple-stress }}^{\text {xz }}} \frac{\partial \phi_{z}}{\partial x}=-M_{x} ; \underbrace{\left(E_{y} I_{z}+4 l_{b 2}^{2} C_{44} H\right)}_{\kappa_{\text {couple-stress }}^{y z}} \frac{\partial \phi_{z}}{\partial y}=-M_{y} .
$$

On the other hand, if we do not take into account the couple-stress effects, the classical bending stiffnesses, quantities $\kappa_{\text {classical }}^{x z}$ and $\kappa_{\text {classical }}^{y z}$, for a homogenous equivalent beam are defined by

$$
\underbrace{\left(E_{x} I_{z}\right)}_{\kappa_{\text {classical }}^{x z}} \frac{\partial \phi_{z}}{\partial x}=-M ; \underbrace{\left(E_{y} I_{z}\right)}_{\kappa_{\text {classical }}^{y z}} \frac{\partial \phi_{z}}{\partial y}=-M .
$$

We here adopt the macroscopic parameter $\mathrm{H}$ and $\mathrm{W}$ equal to 4 and $20 \mathrm{~mm}$ for the auxetic thickness and width, respectively. The beam is subjected to a pure bending with a uniform applied bending moment. The differences between the bending rigidities of the homogenized beam are quantified based on the following variable:

$$
\% \text { diff }=\frac{\kappa_{\text {couple-stress }}-\kappa_{\text {classical }}}{\kappa_{\text {classical }}} \times 100
$$

which represents the gain in flexural rigidity due to the couple-stress effects. It is noticed that the gain in bending rigidity is important compared to a classical beam (without couple-stress effects), since the gain in rigidity is $61 \%$ for bending in the $x-z$ plane and $58 \%$ for bending in the $y-z$ plane.

\section{5 | CONCLUSIONS}

The present work advances a topology optimization based method to conceive optimal auxetic structures presenting an optimal average in-plane Poisson's ratio, relying on the topological derivative. Due to the adopted function to be minimized, the obtained optimal microstructure shows an orthotropic but non isotropic behavior. The auxetic nature of the computed microstructures has been demonstrated by both numerical and real experiments; the numerical evolution of the deformation of the microstructure highlights pronounced rotation effects which motivate the choice of a couple stress effective continuum at the macroscopic level. The effective mechanical properties of auxetic structures have been computed in the framework of couple stress elasticity, in order to evaluate both their in-plane properties and their bending moduli. The classical moduli are found to be independent of the size of the selected window of analysis, whereas the nonclassical (bending) moduli are size-dependent. Measurements performed over samples produced by additive printing chosen large enough to be representative provide in-plane Poisson's ratio close to that computed by numerical experiments and obtained by topology optimization. The concepts and methods advanced in this contributions have the advantage to be systematic and are thus potentially of great interest to design microstructures satisfying other requirements and unusual mechanical properties.

\section{REFERENCES}

[1] S. Amstutz, S. M. Giusti, A. A. Novotny, E. A. de Souza Neto, Topological derivative for multi-scale linear elasticity models applied to the synthesis of microstructures, Int. J. Numer. Methods Eng. 2010, 84, 733.

[2] M. P. Bendsøe, N. Kikuchi, Generating optimal topologies in structural design using an homogenization method, Comput. Methods Appl. Mech. Eng. 1988, 71, 197.

[3] M. P. Bendsøe, O. Sigmund, Topology Optimization. Theory, Methods and Applications, Springer-Verlag, Berlin 2003.

[4] L. Cabras, M. Brun, A class of auxetic three-dimensional lattices, J. Mech. Phys. Solids 2016, 91, 56.

[5] S. Czarnecki, P. Wawruch, The emergence of auxetic material as a result of optimal isotropic design, Phys. Status Solidi $2015,252,1620$.

[6] H. A. Eschenauer, V. V. Kobelev, A. Schumacher, Bubble Method for topology and Shape Optimization, American Mathematical Society, Providence 2010.

[7] S. M. Giusti, A. A. Novotny, E. A. de Souza Neto, Sensitivity of the macroscopic response of elastic microstructures to the insertion of inclusions, in Proceeding of the Royal Society A: Mathematical, Physical and Engineering Sciences 2010, Vol. 466, pp. 1703-1723.

[8] S. M. Giusti, A. A. Novotny, E. A. de Souza Neto, R. A. Feijóo, Sensitivity of the macroscopic elasticity tensor to topological microstructural changes, J. Mech. Phys. Solids 2009, 57, 555. 
[9] I. Goda, R. Rahouadj, J. F. Ganghoffer, Size dependent static and dynamic behavior of trabecular bone based on micromechanical models of the trabecular bone architecture, Int. J. Eng. Sci. 2013, 72, 53.

[10] I. Goda, R. Rahouadj, J. F. Ganghoffer, A 3d elastic micropolar model of vertebral trabecular bone from lattice homogenization of the bone microstructure, Biomech. Model. Mechanobiol. 2014, 13, 53.

[11] X. Huang, A. Radman, Y. M. Xie, Topological design of microstructures of cellular materials for maximum bulk or shear modulus, Comput. Mater. Sci. 2011, 50, 1861.

[12] R. Kureta, Y. Kanno, A mixed integer programming approach to designing periodic frame structures with negative poissons ratio, Eng. Optim. 2014, 15, 773 .

[13] R. Lakes, Deformation mechanisms in negative poissons ratio materials: structural aspects, J. Mater. Sci. 1991, $26,2287$.

[14] R. Lakes, Experimental Methods for Study of Cosserat Elastic Solids and Other Generalized Elastic Continua (Ed.: H-B Muhlhaus) Continuum Models for Materials with Microstructure, Wiley, New Yok 1995, pp. 1-22.

[15] G. W. Milton, Composite materials with poissons ratios close to -1, J. Mech. Phys. Solids 1992, 40, 1105.

[16] M. Mir, M. N. Ali, J. Sami, U. Ansari, Review of mechanics and applications of auxetic structures, Adv. Mater. Sci. Eng. $2014,1$.

[17] A. A. Novotny, J. Sokołowski, Topological Derivatives in Shape Optimization, Interaction of Mechanics and Mathematics, Springer-Verlag, Berlin, Heidelberg 2013.

[18] D. Overaker, A. Cuitino, N. Langrana, Elastoplastic micromechanical modeling of two-dimensional irregular convex and nonconvex (re-entrant) hexagonal foams, J. Appl. Mech. 1998, 65, 748.

[19] A. Radman, X. Huang X, Y. Xie, Topological optimization for the design of microstructures of isotropic cellular materials, Eng. Optim. 2013, 45, 1331.

[20] E. Sanchez-Palencia, Non-Homogeneous Media and Vibration Theory, Lecture Notes in Physics, Vol. 127, Springer-Verlag, Berlin 1980.

[21] A. Sapdoni, M. Ruzzene, Elasto-static micropolar behavior of a chiral auxetic lattice, J. Mech. Phys. Solids 2012, 60, 156.

[22] O. Sigmund, Materials with prescribed constitutive parameters: an inverse homogenization problem, Int. J. Solids Struct. $1994,31,2313$.

[23] J. Sokołowski, A. Zochowski, On the topological derivative in shape optimization, SIAM J. Control Optim. 1999, $37,1251$.

[24] I. Özdemir, Topological derivative based optimization of 3D porous elastic microstructures, Comput. Mater. Sci. $2014,81,319$.

[25] P. Vogiatzis, S. Chen, X. Wang, T. Li, L. Wang, opology optimization of multi-material negative poissons ratio metamaterials using a reconciled level set method, Comput. Aided Des. 2017, 83, 15.

[26] F. Wang, O. Sigmund, J. S. Jensen, Design of materials with prescribed nonlinear properties, J. Mech. Phys. Solids 2014, 69 , 156.

[27] Y. Wang, J. Gao, Z. Luo, T. Brown, N. Zhang, Level-set topology optimization for multimaterial and multifunctional mechanical metamaterials, Eng. Optim. 2017, 49, 22.

[28] Y. Wang, Z. Luo, N. Zhang, Z. Kang, Topological shape optimization of microstructural metamaterials using a level set method, Comput. Mater. Sci. 2014, 87, 178.

[29] Z. P. Wang, L. H. Poh, J. Dirrenberger, Y. Zhu, S. Forest, Isogeometric shape optimization of smoothed petal auxetic structures via computational periodic homogenization, Comput. Methods Appl. Mech. Eng. 2017, 323, 250.

[30] W. Yang, Z. M. Li, W. Shi, B. H. Xie, M. B. Yang, Review on auxetic materials, J. Mater. Sci. 2004, $39,3269$.

How to cite this article: Ganghoffer JF, Goda I, Novotny AA, Rahouadj R, Sokolowski J. Homogenized couple stress model of optimal auxetic microstructures computed by topology optimization. Z Angew Math Mech. 2018;98:696-717. https://doi.org/10.1002/zamm.201700154 